\title{
Using color histogram normalization for recovering chromatic illumination-changed images
}

\author{
Soo-Chang Pei, Ching-Long Tseng, and Chai-Che Wu \\ Department of Electrical Engineering, National Taiwan University, Taipei, Taiwan 10617
}

Received August 7, 2000; accepted March 22, 2001; revised manuscript received April 27, 2001

\begin{abstract}
We propose a novel image-recovery method using the covariance matrix of the red-green-blue (R-G-B) color histogram and tensor theories. The image-recovery method is called the color histogram normalization algorithm. It is known that the color histograms of an image taken under varied illuminations are related by a general affine transformation of the $\mathrm{R}-\mathrm{G}-\mathrm{B}$ coordinates when the illumination is changed. We propose a simplified affine model for application with illumination variation. This simplified affine model considers the effects of only three basic forms of distortion: translation, scaling, and rotation. According to this principle, we can estimate the affine transformation matrix necessary to recover images whose color distributions are varied as a result of illumination changes. We compare the normalized color histogram of the standard image with that of the tested image. By performing some operations of simple linear algebra, we can estimate the matrix of the affine transformation between two images under different illuminations. To demonstrate the performance of the proposed algorithm, we divide the experiments into two parts: computer-simulated images and real images corresponding to illumination changes. Simulation results show that the proposed algorithm is effective for both types of images. We also explain the noise-sensitive skew-rotation estimation that exists in the general affine model and demonstrate that the proposed simplified affine model without the use of skew rotation is better than the general affine model for such applications. () 2001 Optical Society of America
\end{abstract}

OCIS codes: $330.1690,100.3020,100.2960$.

\section{INTRODUCTION}

In recent years the role of color is becoming important in computer vision, as many researchers have used color information to recognize objects. ${ }^{1,2}$ The color of an object varies with changes in illumination source, illumination geometry, viewing angle, and miscellaneous sensor parameters. $^{3}$ But in pattern-recognition applications, researchers usually use the recovery of the color of the object under standard illumination as a template for recognition. The phenomenon of steady spectral color response under different illuminations is called color constancy. ${ }^{4}$ In other words, the color constancy problem consists of removing the illumination-source color to obtain the object's pure surface-reflectance color. ${ }^{5}$ Color constancy algorithms can play a major role in image retrieval from image databases.

Early research on computational color constancy centered on recovering an illumination-invariant surface description at each image location. Two common assumptions in this approach are that the spectral reflectance of the surface is given or that the weighted average of the spectral reflectance in the scene is known. ${ }^{6}$ Another common assumption is that highlights of the dielectric materials can be observed, which contain illumination spectral properties illumination that are useful in color constancy work. ${ }^{7,8}$ Although worth mentioning, these assumptions are not valid for all situations. Consequently, Maloney and Wandell proposed the first computational color constancy algorithm that did not require the above assumptions. ${ }^{4}$ Using the linear surface-reflectance model, they computed color-constant surface descriptors for cases in which photoreceptors have more classes than degrees of freedom. Their results are significant for subsequent research on color constancy.

The color image histogram distribution of an object has been used in pattern recognition for many years. For example, multiband images that carry a large quantity of information about a scene are commonly used in remotesensing applications. In addition, Swain and Ballard proposed an algorithm called color indexing, ${ }^{1}$ which uses the color histogram intersection of the object to compare observed histograms of many different kinds of objects directly from a large database over a range of conditions. The technique does not use any geometric information of objects, but color distributions are often sensitive to illumination changes. That is, the method is feasible only in environments where illumination is not allowed to vary much.

Later, Funt and Finlayson introduced a new algorithm called color-constant color indexing ${ }^{2}$ to approach the above-mentioned problem. The algorithm matches the distributions of color ratios in an illumination-varying environment. They proved that under the coefficient model for sensor response, the distributions of color ratios are illumination invariant. Although the method performs far better than color indexing when illumination color is allowed to change, it still has significant restrictions. For example, color ratios are often sensitive to low-intensity noise levels.

Slater and Healey developed a color neighborhood representation for illumination-invariant matching. ${ }^{9}$ However, using such the representation requires a suitable 
search-and-projection method for indexing. The drawback of the algorithm is that it requires a large amount of storage space and expensive comparisons for representing objects by using distributions or definite structure. Further, Healey and Slater tried to select a vector of six color distribution invariants to recognize objects on a mediumsized database. ${ }^{10-12}$ These color distribution invariants have been demonstrated and used as a feature for image retrieval.

In this paper we propose a novel algorithm that uses color histogram normalization to recover images. Using the statistical properties of color distributions to normalize the color histogram of images, we then derive, by basic linear algebra, the affine transform corresponding to the illumination change. This paper is organized as follows. In Section 2 we review the modeling of color representation. The proposed algorithms for image recovery are described in Section 3. In Section 4 we present the experimental results and discussion of the real natural images. Our conclusions are presented in Section 5.

\section{MODELING COLOR REPRESENTATION}

From Refs. 9-11 we know that $n$ sensor measurements are observed by a color imaging system at each image location $(x, y)$, given by

$$
\rho_{i}(x, y)=\int_{\lambda} l(\lambda) s(x, y, \lambda) f_{i}(\lambda) \mathrm{d} \lambda, \quad 1 \leqslant i \leqslant n,
$$

where $\lambda$ indicates wavelength, $l(\lambda)$ is the spectral power distribution of the scene illumination, $s(x, y, \lambda)$ is the spectral reflectance of the surface, and $f_{i}(\lambda)$ is the spectral sensitivity of the $i$ th sensor class; $n$ is usually 3 , and $f_{1}(\lambda), f_{2}(\lambda)$, and $f_{3}(\lambda)$ represent the spectral response of the red, green, and blue sensing elements for a redgreen-blue $(\mathrm{R}-\mathrm{G}-\mathrm{B})$ color system. The integration is over the entire range of visible wavelengths.

However, the surface reflectance $s(x, y, \lambda)$ can be approximated by the weighted sum of a series of fixed basis functions,

$$
s(x, y, \lambda)=\sum_{j=1}^{m} \sigma_{j}(x, y) S_{j}(\lambda)
$$

where $S_{j}(\lambda)$ are a set of $m$ fixed basis functions and $\sigma_{j}(x, y)$ are the surface weighting coefficients corresponding to location. When the effect of human visual sensitivity is included, the linear model approximations provide excellent fits of measure (at 0.9890 accuracy) to the empirical data for $m \geqslant 3,{ }^{13,14}$ and such approximations have been applied extensively. ${ }^{4,15,16}$ Combining Eqs. (1) and (2), we can find that

$$
\rho(x, y)=\mathbf{A} \sigma(x, y),
$$

where $\rho(x, y)=\left[\rho_{1}(x, y), \rho_{2}(x, y), \ldots, \rho_{n}(x, y)\right]^{T}, \quad$ denotes the column vector of sensor measurements, $\sigma(x, y)=\left[\sigma_{1}(x, y), \sigma_{2}(x, y), \ldots, \sigma_{m}(x, y)\right]^{T}$ denotes the column vector of the fixed basis function weights, and $\mathbf{A}$ is an $n \times m$ matrix with entries

$$
\mathbf{A}_{i j}=\int l(\lambda) S_{j}(\lambda) f_{i}(\lambda) \mathrm{d} \lambda .
$$

Obviously, A depends on the light spectrum wavelength $\lambda$ but is independent of the image location $(x, y)$. In most cases, $m$ is equal to $n$.

Consider color images taken of the same planar surface under different illuminations $l(\lambda)$ and $\tilde{l}(\lambda)$; then the two images are represented as

$$
\rho(x, y)=\mathbf{A} \sigma(x, y), \quad \tilde{\rho}(x, y)=\tilde{\mathbf{A}} \sigma(x, y) .
$$

Assuming that $\mathbf{A}$ and $\tilde{\mathbf{A}}$ corresponding to $l(\lambda)$ and $\tilde{l}(\lambda)$ are nonsingular matrices, one can then show the relationship between the two images by using Eq. (5),

$$
\tilde{\rho}(x, y)=\mathbf{M} \rho(x, y),
$$

where $\mathbf{M}=\tilde{\mathbf{A}} \mathbf{A}^{-1}$.

Let $H(\rho)$ and $H(\tilde{\rho})$ be $n$-dimensional color histograms corresponding to the same surface imaged under different illuminations $l(\lambda)$ and $\widetilde{l}(\lambda)$. From Eq. (6) we can find that

$$
H(\tilde{\rho})=H(\mathbf{M} \rho) .
$$

Thus the three-dimensional (3D) color histograms are related by an affine transformation of the coordinates under changing illumination. Note that Eq. (7) is correct only if no reduction by quantization is done.

\section{THE PROPOSED ALGORITHM FOR IMAGE RECOVERY}

We describe a method to normalize a color histogram with respect to translation, scaling, and rotation in the $3 \mathrm{D}$ color spaces, which result from a change in illumination color. We must emphasize that although a general affine transformation can be separated into five basic forms of distortion-translation, scaling, rotation, skew, and shearing, ${ }^{17}$ - we claim that the effects of translation, scaling, and rotation dominate the distortion of the color histogram and that the effects of skew and shearing can be neglected.

Why do we make this claim? Because in considering the distorted color histogram resulting from a change in illumination color, we find that the histogram's shape does not change very much that it represents there is little effect of skew and shearing. On the other hand, when the image is taken under lighter illuminant the range of the color histogram is larger, indicating an effect of scaling. Moreover, with a green illuminant, the position and shape of the distribution are deflected slightly toward the $\mathrm{G}$ axis, indicating translation and rotation effects. Furthermore, extensive experimental results demonstrate that the average peak signal-to-noise ratio ( $\overline{\mathrm{PSNR}}$ ) is quite low when the color histogram normalization algorithm is considered in the general affine model; this result is not as good as the result from the simplified affine model with respect only to translation, scaling, and rotation. Thus we can come to the reasonable conclusion that the general affine model is not appropriate for practical application of the distorted color histogram resulting from a change in illumination color. Therefore we propose a simplified affine model with respect to translation, 
scaling, and rotation for such applications. A detailed explanation and proof from actual experiments will be given in Section 4.

Note that there have been two previous approaches to rotation for color histograms: (1) color rotation in RGB and (2) the adaptation of the whitening transform. ${ }^{18}$ In these approaches it was proposed that the color vectors are expected on the gray axis in the color system; if it they are not there, the algorithm will be rotated to this axis. However, we want to emphasize that the "rotation" of our proposed algorithm is performed in order to transform the color histogram into a normalized form; the meaning of rotation is quite different from that in Ref. 18 .

A. Color (R-G-B) Histogram Normalization Algorithm A normalization algorithm has been developed that transforms a two-dimensional (2D) pattern into its normalized form such that it is invariant to translation, scaling, rotation, and skew. ${ }^{19}$ In this section we extend the normalization algorithm to $3 \mathrm{D}$ color histograms in R-G-B color space. Here we will consider only three basic forms of the color histogram's distortion: translation, rotation, and scaling.

\section{Feature Extraction}

Let $p(r, g, b)$ denote the color histogram's signature at the location $(r, g, b)$ in $3 \mathrm{D}$ color space. For example,

$$
p(r, g, b)= \begin{cases}1 & \text { if the color exists } \\ 0 & \text { if the color does not exist }\end{cases}
$$

The probability density function $f(r, g, b)$ can be found as

$$
f(r, g, b)=\frac{p(r, g, b)}{\int_{\Omega} p(r, g, b) \mathrm{d} V},
$$

where $\Omega$ is the $3 \mathrm{D}$ color space that we consider and $\mathrm{d} V$ is an infinitesimal volume around $(r, g, b)$. Hence the mean vector $\mathbf{c}$ for the color histogram center is

$$
\begin{aligned}
& \mathbf{c}=\left[\begin{array}{lll}
C_{r} & C_{g} & C_{b}
\end{array}\right]^{T}, \\
& C_{r}=\int_{\Omega} r f(r, g, b) \mathrm{d} V, \\
& C_{g}=\int_{\Omega} g f(r, g, b) \mathrm{d} V, \\
& C_{b}=\int_{\Omega} b f(r, g, b) \mathrm{d} V,
\end{aligned}
$$

and, letting $\mu_{i j k}$ denote the joint central moment, we get

$$
\begin{aligned}
\mu_{i j k} & =E\left[\left(r-C_{r}\right)^{i}\left(g-C_{g}\right)^{j}\left(b-C_{b}\right)^{k}\right] \\
& =\int_{\Omega}\left(r-C_{r}\right)^{i}\left(g-C_{g}\right)^{j}\left(b-C_{b}\right)^{k} f(r, g, b) \mathrm{d} V .
\end{aligned}
$$

So we can define the $3 \mathrm{D}$ joint central-moments covariance matrix $\mathbf{M}$ as

$$
\mathbf{M}=\left[\begin{array}{lll}
\mu_{200} & \mu_{110} & \mu_{101} \\
\mu_{110} & \mu_{020} & \mu_{011} \\
\mu_{101} & \mu_{011} & \mu_{002}
\end{array}\right] .
$$

Similarly to the 2D cases in Ref. 19 in the object space, we may formulate all the distortions by a general affine transformation in $3 \mathrm{D}$ color space:

$$
\left(\begin{array}{c}
u \\
v \\
w
\end{array}\right)=\left[\begin{array}{lll}
a_{11} & a_{12} & a_{13} \\
a_{21} & a_{22} & a_{23} \\
a_{31} & a_{32} & a_{33}
\end{array}\right]\left(\begin{array}{l}
r \\
g \\
b
\end{array}\right)+\left(\begin{array}{l}
b_{1} \\
b_{2} \\
b_{3}
\end{array}\right),
$$

which written in vector form is

$$
\mathbf{u}=\mathbf{A r}+\mathbf{b} \text {. }
$$

Worth mentioning is that the well-defined covariance matrix $\mathbf{M}$ also satisfies theorem 1 in Ref. 19, in either $2 \mathrm{D}$ or $3 \mathrm{D}$ cases. We describe it as theorem 1 here.

Theorem 1: Let $\mathbf{M}$ be the covariance matrix of the original color histogram and $\mathbf{M}^{\prime}$ be the covariance matrix of the affine color histogram in $3 \mathrm{D}$ color spaces. Then matrices $\mathbf{M}$ and $\mathbf{M}^{\prime}$ are related as

$$
\mathbf{M}^{\prime}=\mathbf{A M A}^{T}
$$

where

$$
\mathbf{A}=\left[\begin{array}{lll}
a_{11} & a_{12} & a_{13} \\
a_{21} & a_{22} & a_{23} \\
a_{31} & a_{32} & a_{33}
\end{array}\right]
$$

\section{Algorithm of Normalization That Will Not Deform} the Color Histogram's Shape

Now we describe how to normalize the color histogram. This procedure contains four major steps:

1. Computing the covariance matrix $\mathbf{M}$ of the color histogram.

2. Finding out the eigenvalues and eigenvectors of $\mathbf{M}$ and then determining the exact eigenvectors that will not cause the reflection of the normalized color histogram.

3. Aligning the coordinates with the exact eigenvectors.

4. Rescaling the new coordinates by using the corresponding eigenvalues of $\mathbf{M}$.

Step 1. Computing the Covariance Matrix M. The purpose of the process is to rotate and rescale the color histogram such that the transformed color histogram is invariant under translation, rotation, and scaling but not to deform the color histogram's shape. By Eq. (14), we may calculate the elements of $\mathbf{M}$.

Step 2. Determining the Exact Eigenvectors. We can easily compute the eigenvalues and eigenvectors of $\mathbf{M}$. Let $\mathbf{e}_{1}$ be the eigenvector associated with eigenvalue $\lambda_{1}$, $\mathbf{e}_{2}$ be the eigenvector associated with eigenvalues $\lambda_{2}$, and $\mathbf{e}_{3}$ be the eigenvector associated with eigenvalue $\lambda_{3}$, where $\lambda_{1} \leqslant \lambda_{2} \leqslant \lambda_{3}$.

Since $\mathbf{M}$ is symmetric, the eigenvectors $\mathbf{e}_{1}, \mathbf{e}_{2}, \mathbf{e}_{3}$ are orthonormal to one another. To avoid the reflection of the normalized color histogram, we must choose the direction of $\mathbf{e}_{3}$ according to the direction of the cross product of $\mathbf{e}_{1}$ and $\mathbf{e}_{2}$ : 


$$
\mathbf{e}_{3}=\mathbf{e}_{1} \times \mathbf{e}_{2} \text {. }
$$

After the arrangement of Eq. (20), there are still four possible combinations of the three eigenvectors:

$$
\begin{aligned}
& \left(\begin{array}{lll}
\mathbf{e}_{1} & \mathbf{e}_{2} & \mathbf{e}_{3}
\end{array}\right), \quad\left(\begin{array}{lll}
\mathbf{e}_{1} & -\mathbf{e}_{2} & -\mathbf{e}_{3}
\end{array}\right), \\
& \left(\begin{array}{lll}
-\mathbf{e}_{1} & \mathbf{e}_{2} & -\mathbf{e}_{3}
\end{array}\right), \quad\left(\begin{array}{lll}
-\mathbf{e}_{1} & -\mathbf{e}_{2} & \mathbf{e}_{3}
\end{array}\right) .
\end{aligned}
$$

We must determine a coordinate system from these four possible cases that will not cause reflection of the normalized color histogram as we rotate the color histogram to the new coordinate.

To choose one proper coordinate system that is invariant under translation, rotation, and scaling, we analyze some properties of the eigenvectors of $\mathbf{M}$.

Case 1: Translation. For translation, the covariance matrix $\mathbf{M}^{\prime}$ of the translated color histogram is equal to the covariance matrix $\mathbf{M}$ of the original color histogram. Therefore their eigenvectors and eigenvalues are the same. Thus aligning the coordinates with the eigenvectors if the distortion is due to translation will cause no reflection.

Case 2: Scaling. For scaling, $\mathbf{A}=a \mathbf{I}$, where $a$ is the scaling factor. By theorem 1,

$$
\mathbf{M}^{\prime}=\mathbf{A M A}^{T}=a^{2} \mathbf{M} .
$$

Thus the eigenvalues of $\mathbf{M}^{\prime}=\left(\begin{array}{lll}\lambda_{1}^{\prime} & \lambda_{2}^{\prime} & \lambda_{3}^{\prime}\end{array}\right)$ $=\left(\begin{array}{lll}a^{2} \lambda_{1} & a^{2} \lambda_{2} & a^{2} \lambda_{3}\end{array}\right)$, but they have the same eigenvectors. Thus aligning the coordinates with the eigenvectors if the distortion is due to scaling will cause no reflection.

Case 3: Rotation. For the case of rotation, $\mathbf{A}$ is an orthogonal matrix; i.e.,

$$
\mathbf{A}^{T}=\mathbf{A}^{-1}
$$

By theorem 1,

$$
\mathbf{M}^{\prime}=\mathbf{A M A}^{T}=\mathbf{A M A}^{-1},
$$

so $\mathbf{M}^{\prime}$ is similar to $\mathbf{M}$; in other words, $\mathbf{M}^{\prime}$ and $\mathbf{M}$ have the same eigenvalues. But because their eigenvectors are different, the relation between the two sets of eigenvectors is

$$
\mathbf{E}^{\prime}=\mathbf{A}^{-1} \mathbf{E} \text { or } \mathbf{E}^{\prime}=-\mathbf{A}^{-1} \mathbf{E},
$$

where

$$
\mathbf{E}=\left(\begin{array}{c}
\mathbf{e}_{1}^{T} \\
\mathbf{e}_{2}^{T} \\
\mathbf{e}_{3}^{T}
\end{array}\right), \quad \mathbf{E}^{\prime}=\left(\begin{array}{c}
\mathbf{e}_{1}^{\prime T} \\
\mathbf{e}_{2}^{\prime T} \\
\mathbf{e}_{3}^{\prime T}
\end{array}\right) .
$$

If $\mathbf{E}^{\prime}=\mathbf{A}^{-1} \mathbf{E}$, we can get the same orientation after we rotate the two color histograms individually according to their eigenvectors. If $\mathbf{E}^{\prime}=-\mathbf{A}^{-1} \mathbf{E}$, the normalized color histograms are mirror symmetric to each other. In addition to the four possible combinations of these eigenvectors in Eq. (21), it is impossible to get the same orientation of normalized color histograms without modification of these eigenvectors.

In the following, we discuss how to choose the exact eigenvectors to get the same orientation of normalized color histograms. According to Eq. (21), the four possible rotation matrices are

$$
\begin{aligned}
& \mathbf{E}_{1}=\left(\begin{array}{c}
\mathbf{e}_{1}^{T} \\
\mathbf{e}_{2}^{T} \\
\mathbf{e}_{3}^{T}
\end{array}\right)=\left[\begin{array}{lll}
e_{1 r} & e_{1 g} & e_{1 b} \\
e_{2 r} & e_{2 g} & e_{2 b} \\
e_{3 r} & e_{3 g} & e_{3 b}
\end{array}\right], \\
& \mathbf{E}_{2}=\left(\begin{array}{c}
\mathbf{e}_{1}^{T} \\
-\mathbf{e}_{2}^{T} \\
-\mathbf{e}_{3}^{T}
\end{array}\right)=\left[\begin{array}{ccc}
e_{1 r} & e_{1 g} & e_{1 b} \\
-e_{2 r} & -e_{2 g} & -e_{2 b} \\
-e_{3 r} & -e_{3 g} & -e_{3 b}
\end{array}\right], \\
& \mathbf{E}_{3}=\left(\begin{array}{c}
-\mathbf{e}_{1}^{T} \\
\mathbf{e}_{2}^{T} \\
-\mathbf{e}_{3}^{T}
\end{array}\right)=\left[\begin{array}{ccc}
-e_{1 r} & -e_{1 g} & -e_{1 b} \\
e_{2 r} & e_{2 g} & e_{2 b} \\
-e_{3 r} & -e_{3 g} & -e_{3 b}
\end{array}\right], \\
& \mathbf{E}_{4}=\left(\begin{array}{c}
-\mathbf{e}_{1}^{T} \\
-\mathbf{e}_{2}^{T} \\
\mathbf{e}_{3}^{T}
\end{array}\right)=\left[\begin{array}{ccc}
-e_{1 r} & -e_{1 g} & -e_{1 b} \\
-e_{2 r} & -e_{2 g} & -e_{2 b} \\
e_{3 r} & e_{3 g} & e_{3 b}
\end{array}\right] .
\end{aligned}
$$

In the normalization algorithm we transform the original color histogram according to one of the four rotational matrices; i.e.,

$$
\left(\begin{array}{l}
r^{\prime} \\
g^{\prime} \\
b^{\prime}
\end{array}\right)=\mathbf{E}_{1}\left(\begin{array}{l}
r-C_{r} \\
g-C_{g} \\
b-C_{b}
\end{array}\right), \quad i \text { in }\{1,2,3,4\} .
$$

Similarly, we apply the tensor theories to normalized color histograms. ${ }^{20}$ First we present the tensor representation of $3 \mathrm{D}$ color space and some theories, and then we apply tensor theories to normalize color histograms. As at the outset of Section 3, we can express the affine transformation as Eq. (16). Therefore the first-order moments of the color histograms are denoted by $\left(\begin{array}{llll}C_{r} & C_{g} & C_{b}\end{array}\right)^{T}$, and the jointly central moments are denoted by $\mu_{i j k}$. If we translate the coordinates to the center of the color histogram, Eq. (16) becomes

$$
\left(\begin{array}{c}
u-C_{u} \\
v-C_{v} \\
w-C_{w}
\end{array}\right)=\left[\begin{array}{lll}
a_{11} & a_{12} & a_{13} \\
a_{21} & a_{22} & a_{23} \\
a_{31} & a_{32} & a_{33}
\end{array}\right]\left(\begin{array}{l}
r-C_{r} \\
g-C_{g} \\
b-C_{b}
\end{array}\right) .
$$

Obviously, Eq. (32) is invariant to translation. The origin of coordinates is assumed at the center of color histogram to simplify notation in the following discussion.

In tensor notation, the coordinate variables are differentiated by an index; i.e., $x^{1}$ here is equivalent to the $r$ coordinate, $x^{2}$ is equivalent to the $g$ coordinate, and $x^{3}$ is equivalent to the $b$ coordinate. Thus Eq. (32) becomes

$$
\left(\begin{array}{l}
y^{1} \\
y^{2} \\
y^{3}
\end{array}\right)=\left[\begin{array}{lll}
A_{1}^{1} & A_{2}^{1} & A_{3}^{1} \\
A_{1}^{2} & A_{2}^{2} & A_{3}^{2} \\
A_{1}^{3} & A_{2}^{3} & A_{3}^{3}
\end{array}\right]\left(\begin{array}{c}
x^{1} \\
x^{2} \\
x^{3}
\end{array}\right)
$$

where

$$
\begin{gathered}
\left(\begin{array}{l}
y^{1} \\
y^{2} \\
y^{3}
\end{array}\right)=\left(\begin{array}{c}
u-C_{u} \\
v-C_{v} \\
w-C_{w}
\end{array}\right), \quad\left(\begin{array}{l}
x^{1} \\
x^{2} \\
x^{3}
\end{array}\right)=\left(\begin{array}{l}
r-C_{r} \\
g-C_{g} \\
b-C_{b}
\end{array}\right), \\
{\left[\begin{array}{lll}
A_{1}^{1} & A_{2}^{1} & A_{3}^{1} \\
A_{1}^{2} & A_{2}^{2} & A_{3}^{2} \\
A_{1}^{3} & A_{2}^{3} & A_{3}^{3}
\end{array}\right]=\left[\begin{array}{lll}
a_{11} & a_{12} & a_{13} \\
a_{21} & a_{22} & a_{23} \\
a_{31} & a_{32} & a_{33}
\end{array}\right] .}
\end{gathered}
$$




$$
y^{i}=A_{j}^{i} x^{j} \quad \text { for } i=1,2,3 .
$$

Hence we may define the inverse affine transformation,

$$
x^{i}=a_{j}^{i} y^{j} \quad \text { for } i=1,2,3,
$$

with the property

$$
A_{j}^{i} a_{k}^{j}=\left\{\begin{array}{ll}
1 & \text { if } i=k \\
0 & \text { if } i \neq k
\end{array}\right\}=\delta_{i k} .
$$

The central moment tensors are defined as

$$
T^{i j k \ldots}=\int_{\Omega} x^{i} x^{j} x^{k} \ldots f\left(x^{1}, x^{2}\right) \mathrm{d} x^{1} \mathrm{~d} x^{2},
$$

where $f\left(x^{1}, x^{2}\right)$ can be regarded as the probability density function as in Eq. (9).

This type of tensor is the case of contravariant tensor operators. Referring to Ref. 19, we get the following theorem:

Theorem 2: If we let

$$
\begin{aligned}
& T^{i j k \ldots}=\int_{\Omega} x^{i} x^{j} x^{k} \ldots f\left(x^{1}, x^{2}\right) \mathrm{d} x^{1} \mathrm{~d} x^{2}, \\
& \bar{T}^{i j k \ldots}=\int_{\Sigma} y^{i} y^{j} y^{k} \ldots f\left(y^{1}, y^{2}\right) \mathrm{d} y^{1} \mathrm{~d} y^{2},
\end{aligned}
$$

if $\left(x^{i} x^{j} x^{k}\right)$ and $\left(y^{i} y^{j} y^{k}\right)$ are related as tensors; i.e.,

$$
y^{i} y^{j} y^{k} \ldots=A_{l}^{i} A_{m}^{j} A_{n}^{k} \ldots x^{l} x^{m} x^{n} \ldots
$$

then $\left(T^{i} T^{j} T^{k}\right)$ and $\left(\bar{T}^{i} \bar{T}^{j} \bar{T}^{k}\right)$ are also related as tensors; i.e.,

$$
\bar{T}^{i} \bar{T}^{j} \bar{T}^{k} \ldots=A_{l}^{i} A_{m}^{j} A_{n}^{k} \ldots T^{l} T^{m} T^{n} \ldots
$$

As mentioned above, we may determine which is the exact rotational matrix. In Eq. (31) the relation between the transformed and the original tensor is

$$
\bar{T}^{i j k}(\mathbf{A})=A_{l}^{i} A_{m}^{j} A_{n}^{k} T^{l m n},
$$

where $A_{j}^{i}$ is the element $(i, j)$ of matrix $\mathbf{A}$; for example, when we calculate the transformed tensor $\bar{T}^{111}$ for $i=j$ $=k=1$ in Eq. (43), $A_{j}^{1}$ are the elements of the first row of the matrix A. On the other hand, the calculation of $T^{l m n}$ can be regarded as the calculation of $3 \mathrm{D}$ jointly central moment as in Eq. (14) according to the definition of tensors such as Eqs. (39) and (40).

Substituting the rotation matrix E of Eqs. (27)-(30) into Eq. (43), we get the relations

$$
\begin{aligned}
& \bar{T}^{111}\left(\mathbf{E}_{1}\right)=\bar{T}^{111}\left(\mathbf{E}_{2}\right)=-\bar{T}^{111}\left(\mathbf{E}_{3}\right)=-\bar{T}^{111}\left(\mathbf{E}_{4}\right), \\
& \bar{T}^{222}\left(\mathbf{E}_{1}\right)=-\bar{T}^{222}\left(\mathbf{E}_{2}\right)=\bar{T}^{222}\left(\mathbf{E}_{3}\right)=-\bar{T}^{222}\left(\mathbf{E}_{4}\right) .
\end{aligned}
$$

First choosing $\bar{T}^{111}>0$, we may differentiate $\mathbf{E}_{1} \quad \mathbf{E}_{2}$ from $\mathbf{E}_{3} \quad \mathbf{E}_{4}$. Furthermore, setting $\bar{T}^{222}>0$, we may differentiate $\mathbf{E}_{1}$ from $\mathbf{E}_{2}$.

Therefore we can uniquely determine a rotational matrix that will not cause reflection of the normalized color histograms. In addition, this choice can also solve the mirror phenomenon in Eq. (25). In conclusion, the exact eigenvectors may be found by the criterion
Choose $\mathbf{E}$ in $\left\{\begin{array}{llll}\mathbf{E}_{1} & \mathbf{E}_{2} & \mathbf{E}_{3} & \mathbf{E}_{4}\end{array}\right\}$ such that

$$
\bar{T}^{111}>0 \text { and } \bar{T}^{222}>0 \text { simultaneously. }
$$

Step 3. Aligning the Coordinates with the Exact Eigenvectors. After finding out the exact eigenvectors, we rotate the color histogram to new coordinates:

$$
\left(\begin{array}{l}
r^{\prime} \\
g^{\prime} \\
b^{\prime}
\end{array}\right)=\mathbf{E}\left(\begin{array}{l}
r-C_{r} \\
g-C_{g} \\
b-C_{b}
\end{array}\right) .
$$

Step 4. Rescaling the Coordinates by Using the Eigenvalues of M. With the transformation of Eq. (47), only the scaling distortion remains unsolved. By the previous discussion of case 2 , the eigenvalues of $\mathbf{M}^{\prime}$ is related to $\mathbf{M}$ by

$$
\lambda_{i}^{\prime}=a^{2} \lambda_{i}, \quad i=1,2,3 .
$$

Letting $\lambda=\lambda_{1}+\lambda_{2}+\lambda_{3}$, when we rescale the color histogram by the factor $c / \sqrt{\lambda}$ ( $c$ is constant), the transformed color histogram becomes invariant to scaling.

When we summarize the above discussion, the normalized color histogram may be found by the following transformation:

$$
\left(\begin{array}{l}
r^{\prime \prime} \\
g^{\prime \prime} \\
b^{\prime \prime}
\end{array}\right)=\frac{c}{\sqrt{\lambda}} \mathbf{E}\left(\begin{array}{l}
r-C_{r} \\
g-C_{g} \\
b-C_{b}
\end{array}\right)
$$

Equation (48) seems to be related to the principal components analysis (PCA), which is a powerful statistical tool for feature extraction and data compression. ${ }^{21}$ Although PCA is also an important application of eigenvectors and eigenvalues for finding the directions of greatest variance of a covariance matrix, ${ }^{18,22}$ extra rotation, scaling, and translation with tensor theory are needed for our normalization algorithm. This is the major difference between our algorithm and PCA. On the basis of the color histogram normalization, we can propose a simple, efficient, and illuminant-invariant color image recognition algorithm. We get the normalized color images with a simplified affine model and then extract the block average feature vectors from them to perform color-image recognition very effectively. That is, to average all the pixel values in each block, which are obtained by dividing up the normalized color image into its normalized domains, we can obtain the critical vector $\mathbf{v}$ as a feature to represent this image; it is the block-average feature. Then we use the distance difference $D$ to achieve image recognition, if the difference $D$ is a minimum value between the corresponding critical vector $\mathbf{v}$ of the selected image and the test image critical vector $\mathbf{v}^{\prime}$ in the database. The preliminary experimental results show that the proposed technique is extremely successful; in a database with 10 standard images and 60 illumination-changed test images, the recognition rate of image queries is $91.67 \%$ for the normalized color image divided into $2 \times 2$ blocks, and the recognition rate reaches $100 \%$ for $4 \times 4$ blocks. The specific details of the recognition algorithm are described in Ref. 23. At the same time, the related PCA algorithm is quite efficient for recognizing $3 \mathrm{D}$ objects. ${ }^{5}$ It is of interest that some PCA-related methods are applied in model-based object recognition, ${ }^{24}$ remotely sensed data 
classification, ${ }^{25}$ facial-expression classification, ${ }^{26}$ handwritten kanji recognition, ${ }^{27}$ etc.

\section{Summary of Three-Dimensional Color Histogram} Normalization

The steps of the algorithm are as follows:

(1) Computing the following moments of the original color histogram;

1. Mean vector $\mathbf{c}$ of the center of an color histogram's appearance in 3D color space,

2. Covariance matrix $\mathbf{M}$,

3. Third-order joint central moment $\mu_{i j k}$. M.

(2) Finding the eigenvalues $\lambda_{i}$ and eigenvectors $\mathbf{e}_{i}$ of

(3) According to Eq. (43), computing the tensors $\bar{T}^{111}$ and $\bar{T}^{222}$ :

$$
\begin{aligned}
& \text { If }\left(\bar{T}^{111}<0\right) \text {, then } \mathbf{e}_{1}=-\mathbf{e}_{1} . \\
& \text { If }\left(\bar{T}^{222}<0\right) \text {, then } \mathbf{e}_{2}=-\mathbf{e}_{2} . \\
& \mathbf{e}_{3}=\mathbf{e}_{1} \times \mathbf{e}_{2},
\end{aligned}
$$$$
\text { where } \times \text { denotes cross product. }
$$

(4) The normalized color histogram can be obtained by the following transformation:

$$
\left(\begin{array}{l}
r^{\prime \prime} \\
g^{\prime \prime} \\
b^{\prime \prime}
\end{array}\right)=\frac{c}{\sqrt{\lambda}} \mathbf{E}\left(\begin{array}{l}
r-C_{r} \\
g-C_{g} \\
b-C_{b}
\end{array}\right)
$$

The normalization algorithm has been applied to $3 \mathrm{D}$ objects successfully in computer vision; the specified details are described in Refs. 28 and 29.

\section{B. Color Histogram Normalization Algorithm for Image Recovery}

In this paper we intend to remove the illumination color to obtain the object's pure surface-reflectance color. On the basis of Ref. 10 and the discussion at the beginning of Section 3, we see that there is an important affine property of color distribution change: The effects of translation, scale, and rotation on the appearance of the color histogram depend on the illumination variation. This is true because we assume a finite-dimensional linear surface-reflectance model, changing illumination color to cause a linear transformation of color distributions in $\mathrm{R}-\mathrm{G}-\mathrm{B}$ space. In that case the two $3 \mathrm{D}$ color histograms are related by an affine transformation of the coordinates when the illumination is changed. Therefore the relation between the two color histograms of the original image and the illumination-changed image can be expressed as

$$
\left(\begin{array}{l}
r^{\prime} \\
g^{\prime} \\
b^{\prime}
\end{array}\right)=\left[\begin{array}{lll}
a_{11} & a_{12} & a_{13} \\
a_{21} & a_{22} & a_{23} \\
a_{31} & a_{32} & a_{33}
\end{array}\right]\left(\begin{array}{l}
r \\
g \\
b
\end{array}\right)+\left(\begin{array}{l}
b_{1} \\
b_{2} \\
b_{3}
\end{array}\right) .
$$

By estimating the affine coefficients with the normalization algorithm, we can derive the effects of the different illuminations on the two images. The system block diagram is shown in Fig. 1.

With Eq. (53), the color histogram of the original image is normalized by the transformation

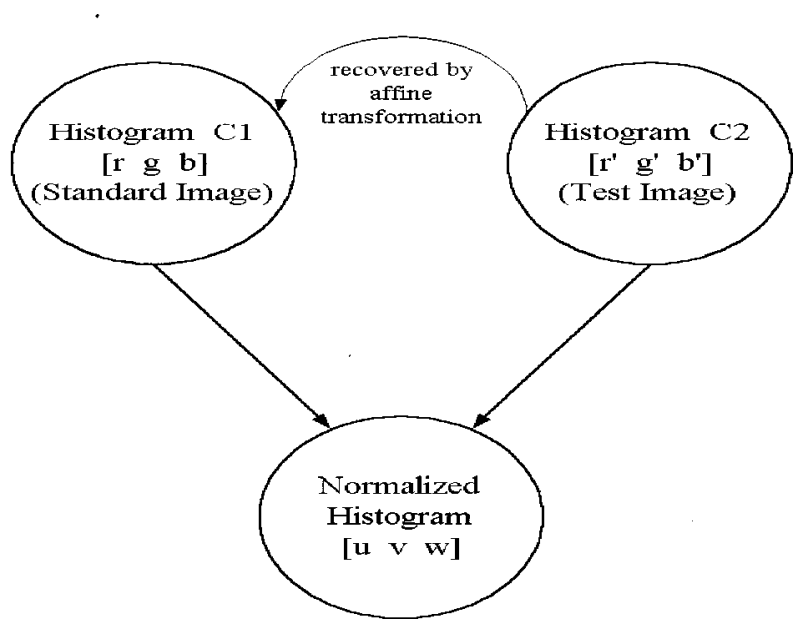

Fig. 1. Diagram of color histogram normalization.

$$
\left(\begin{array}{c}
u \\
v \\
w
\end{array}\right)=\frac{c}{\sqrt{\lambda_{o}}} \mathbf{E}_{o}\left(\begin{array}{l}
r-C_{r} \\
g-C_{g} \\
b-C_{b}
\end{array}\right) .
$$

Similarly, the color histogram of the illumination-changed image is normalized by the transformation

$$
\left(\begin{array}{c}
u \\
v \\
w
\end{array}\right)=\frac{c}{\sqrt{\lambda_{a}}} \mathbf{E}_{a}\left(\begin{array}{l}
r^{\prime}-C_{r}^{\prime} \\
g^{\prime}-C_{g}^{\prime} \\
b^{\prime}-C_{b}^{\prime}
\end{array}\right)
$$

Since the same normalized histograms are obtained, we combine Eqs. (55) and (56).

$$
\left(\begin{array}{l}
r^{\prime}-C_{r}^{\prime} \\
g^{\prime}-C_{g}^{\prime} \\
b^{\prime}-C_{b}^{\prime}
\end{array}\right)=\frac{\sqrt{\lambda_{a}}}{\sqrt{\lambda_{o}}} \mathbf{E}_{a}^{T} \mathbf{E}_{o}\left(\begin{array}{l}
r-C_{r} \\
g-C_{g} \\
b-C_{b}
\end{array}\right) .
$$

Note that $\mathbf{E}_{a}^{T} \mathbf{E}_{o}$ is the rotational matrix between the two histograms, $\sqrt{\lambda_{a}} / \sqrt{\lambda_{o}}$ is the scaling factor, and $C_{r}^{\prime}-C$ in Eq. (10) is the displaced vector between the two histograms. Let

$$
\mathbf{G}=\frac{\sqrt{\lambda_{a}}}{\sqrt{\lambda_{o}}} \mathbf{E}_{a}^{T} \mathbf{E}_{o}
$$

Equation (57) may be rearranged as

$$
\left(\begin{array}{l}
r^{\prime} \\
g^{\prime} \\
b^{\prime}
\end{array}\right)=\mathbf{G}\left(\begin{array}{l}
r \\
g \\
b
\end{array}\right)+\left(\begin{array}{c}
C_{r}^{\prime} \\
C_{g}^{\prime} \\
C_{b}^{\prime}
\end{array}\right)-\mathbf{G}\left(\begin{array}{l}
C_{r} \\
C_{g} \\
C_{b}
\end{array}\right) .
$$

Comparing Eq. (59) with Eq. (54), we can get the affine transform coefficients:

$$
\begin{gathered}
{\left[\begin{array}{lll}
a_{11} & a_{12} & a_{13} \\
a_{21} & a_{22} & a_{23} \\
a_{31} & a_{32} & a_{33}
\end{array}\right]=\mathbf{G},} \\
\left(\begin{array}{l}
b_{1} \\
b_{2} \\
b_{3}
\end{array}\right)=\left(\begin{array}{l}
C_{r}^{\prime} \\
C_{g}^{\prime} \\
C_{b}^{\prime}
\end{array}\right)-\mathbf{G}\left(\begin{array}{l}
C_{r} \\
C_{g} \\
C_{b}
\end{array}\right) .
\end{gathered}
$$

In other words, the color histogram of the original image can be obtained from the color histogram of the illumination-changed image by the following transformation: 


$$
\left(\begin{array}{l}
r \\
g \\
b
\end{array}\right)=\mathbf{G}^{-1}\left(\begin{array}{l}
r^{\prime} \\
g^{\prime} \\
b^{\prime}
\end{array}\right)+\left(\begin{array}{c}
C_{r} \\
C_{g} \\
C_{b}
\end{array}\right)-\mathbf{G}^{-1}\left(\begin{array}{c}
C_{r}^{\prime} \\
C_{g}^{\prime} \\
C_{b}^{\prime}
\end{array}\right) .
$$

\section{EXPERIMENTAL RESULTS AND DISCUSSION}

To demonstrate the proposed color constancy algorithm, we tested seven natural images taken under different illumination conditions. The experiment is divided into two parts in order to test the performance of the proposed algorithm in different circumstances. We chose the initial images under standard illumination as the standard template image for recovering. We obtained the standard template image using our image-acquisition facility with a Sharp VL-H450 DIS Hi8 and an Adobe Premiere 4.0 montage. We emphasize that we use the fullresolution histograms in all the experiments (tristimulus values are all integers ranging between 0 and 255 directly extracted from the source images), and the size of all images is $128 \times 128$.

\section{A. Synthetic Images, Taken under Different Illuminations, Are Simulated by Computer}

1. Experimental Setup

We will use some image-processing software to simulate the illuminant-changed images; the noise in these computer simulation images is usually much less than in the real images. Therefore seven additional images of each picture were fabricated under lighter, darker, red, yellow, green, blue, and magenta by the Paint Shop Pro 5.0 software for several illumination adjustments. The manner of the adjustment is to use the instruction "Adjust" in the "Colors" item with Paint Shop Pro 5.0 to obtain the simulation images. For example, if we want to obtain a simulated image under red illumination, we set the red component to $30 \%$ of the standard template image; the rest of the components are unchanged. Similarly, if we want to obtain the simulated image under darker illumination, we will diminish the three components to $30 \%$ of the standard template image. Tristimulus values should be controlled to avoid clipping in any color band by the software.

\section{Experimental Results}

Next we will show the experimental results of computersimulated images obtained by using Paint Shop Pro 5.0. In Fig. 2, in the original image set [top left (a)-(d)], the upper left figure [label (a)] shows the image with the basket of fruit taken under standard illumination. We regard it as the standard template image. The color histogram of the image is displayed in the upper right figure [label (b)]. Then, using the proposed color histogram normalization algorithm, we show the normalization result of the color histogram in the lower left figure [label (c)]. Similarly, in the set with the computer-simulated image taken under the yellow illumination [top right set (a) (d)], the upper left figure [label (a)] shows the same image but taken under yellow illumination; it is regarded as the test image. The upper right figure [label (b)] and the lower left figure [label (c)] are the color histogram and the normalized one of this test image, respectively. Then, us- ing some linear algebra operations with the two extremely similar normalized patterns of standard image and test image (see Subsection 3.B and the system diagram in Fig. 1), we can obtain a matrix that represents the affine transformation between these two images' histograms. Through the affine transformation, the test image can be recovered to be close to the standard image. The proposed procedure is to use the color histogram normalization algorithm for recovering the illuminationchanged images. The recovered image is shown in the lower right figure [label (d)] of each computer-simulated image set for test images fabricated under yellow, green, and blue by Paint Shop Pro 5.0. Figure 3 consists of images of a famous painting by Manet, "Suonatore di Differaro." These images were fabricated under darker, red, and magenta by Paint Shop Pro 5.0 as the tested images to be recovered. As can be seen, the recovery performance is very good. The peak signal-to-noise ratio (PSNR) value of these recovered images, as shown in Tables 1 and 2 for the basket of fruit and "Suonatore di Differaro," respectively. We define the PSNR as follows:

$$
\begin{aligned}
\text { PSNR_R }= & \sum_{i=1}^{128} \sum_{j=1}^{128}\left[R(i, j)-R^{\prime}(i, j)\right]^{2} /(128 \times 128 \\
& \left.\times \max \_R^{2}\right), \\
\text { PSNR_G }= & \sum_{i=1}^{128} \sum_{j=1}^{128}\left[G(i, j)-G^{\prime}(i, j)\right]^{2} /(128 \times 128 \\
& \left.\times \max \_G^{2}\right), \\
& 128128 \\
\text { PSNR_B }= & \sum_{i=1} \sum_{j=1}\left[B(i, j)-B^{\prime}(i, j)\right]^{2} /(128 \times 128 \\
& \left.\times \max _{-} \mathrm{B}^{2}\right), \\
\overline{\text { PSNR }}= & (\text { PSNR_R }+ \text { PSNR_G }+ \text { PSNR_B }) / 3
\end{aligned}
$$

where PSNR_R, PSNR_G, and PSNR_B denote the PSNR of red, green, and blue bands of the color images respectively, and $\overline{\text { PSNR }}$ denotes the average PSNR value of three bands of the color images, from Eqs. (63)-(66). $R(i, j), G(i, j)$, and $B(i, j)$ represent tristimulus values of the R-G-B color space of the standard template image, and $R^{\prime}(i, j), G^{\prime}(i, j)$, and $B^{\prime}(i, j)$ represent those of the recovered image. Also, $\max \_R, \max _{-} G$, and $\max _{-} B$ indicate the maximum peak value of $R(i, j), G(i, j)$, and $B(i, j)$ individually. From these PSNR tables we can obtain approximately $30-40 \mathrm{~dB}$ average $\overline{\mathrm{PSNR}}$ for computer-simulated images. Results from five other images are not displayed here to save space: "Vaso di Fiori," "Apple and Bottle," "The Musketeer and Pigeon," "Fruits of the Midi," and "The Artist's House at Arles", here we list only the PSNR values of "Vaso di Fiori" in Table 3 for reference.

\section{B. Real Images Taken under Different Illuminations Are Captured by the Camera}

1. Experimental Setup

Figure 4 shows a sketched map of the obtaining of the real images taken under different illuminations. In order to demonstrate the recovery performance for color im- 


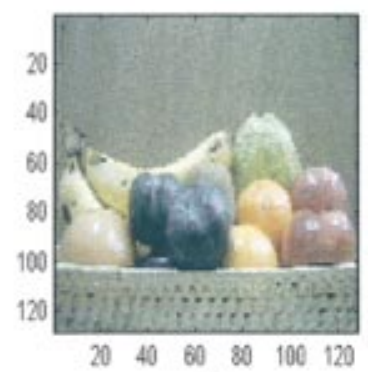

(e)

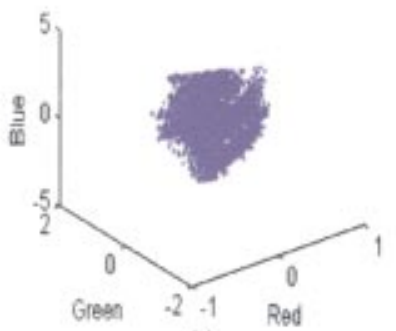

(c)

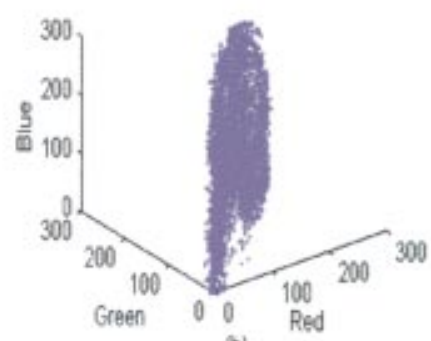

(b)

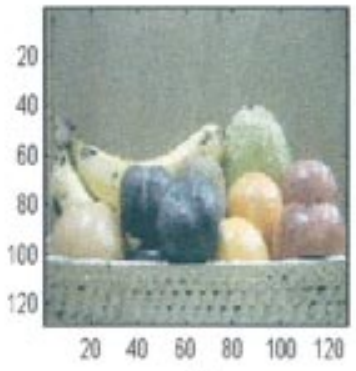

(d)

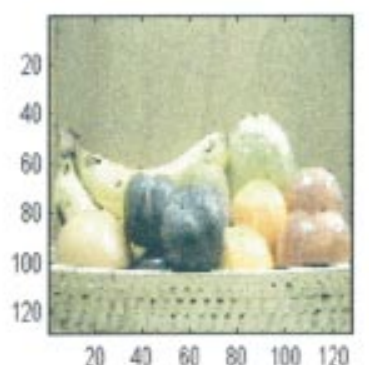

(a)

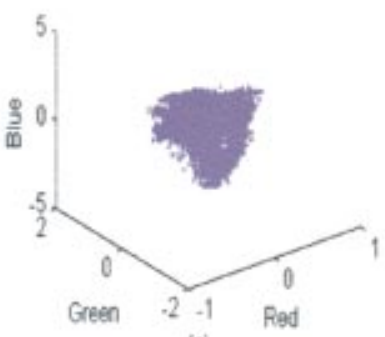

(c)

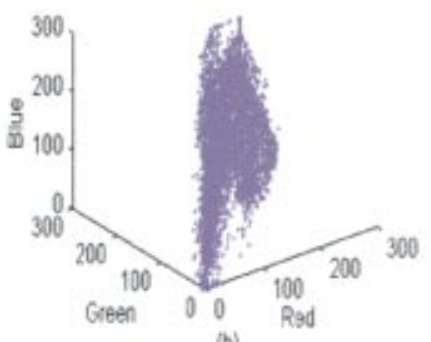

(b)

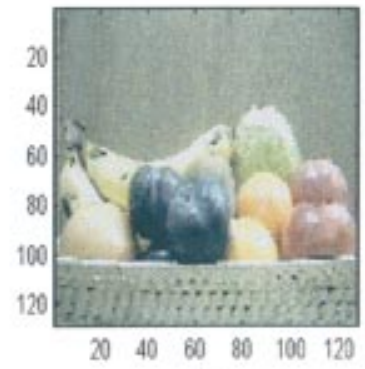

(d)

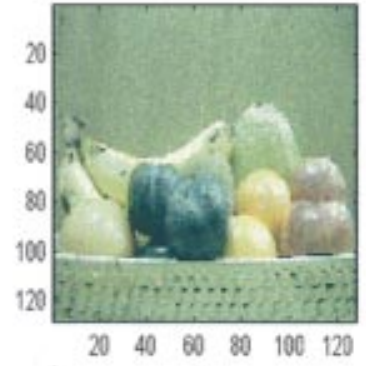

(a)

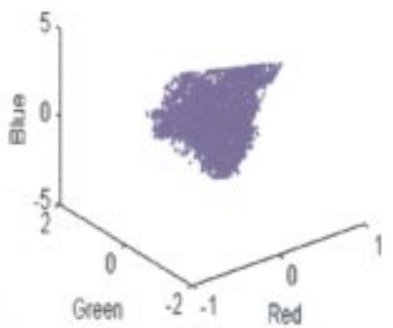

(c)

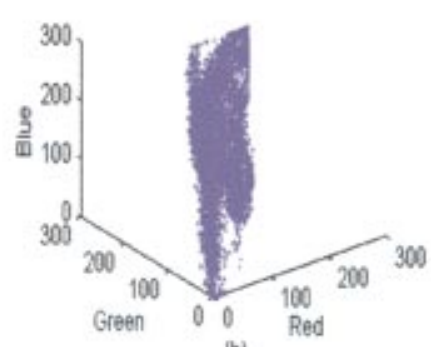

(b)

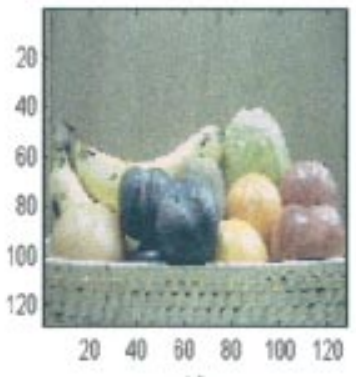

(d)

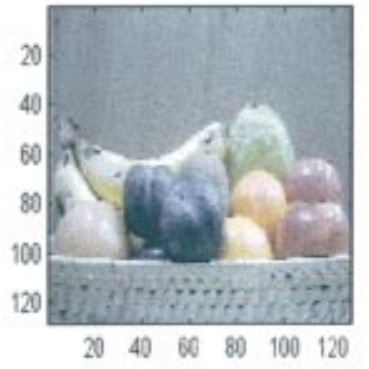

(a)

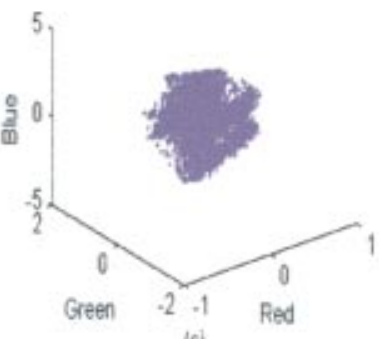

(c)

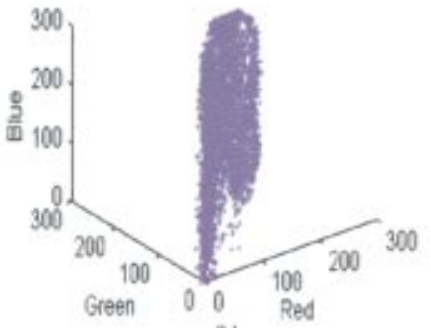

(D)

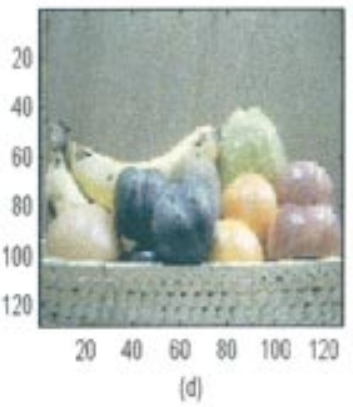

Fig. 2. Basket of fruit, one original (real) image (top left) taken under standard illumination and three computer-simulated images taken under yellow (top right), green (bottom left), and blue (bottom right) illumination. In each set: (b) color histogram of (a), (c) normalized histogram of (b), (d) recovered image by use of the color histogram normalization algorithm.

ages under several illuminants, an additional seven images of each picture were captured under lighter, darker, red, yellow, green, blue, and magenta, by changing the standard source. Similarly, we obtained these real im- ages, using our image-acquisition facility, with a Sharp VL-H450 DIS Hi8 and an Adobe Premiere 4.0 montage. The illumination intensity should be controlled to avoid clipping in any color band. 


\section{Experimental Results}

We now present the experimental results for real images taken under different illuminations. In Fig. 5 the original image set is not shown, because it is exactly the same as in Fig. 2. The top left set (a)-(d) is for the real image of the basket of fruit taken under lighter illumination; the test image is at the upper left [label (a)]. The upper right figure [label (b)] and the lower left figure [label (c)] are the

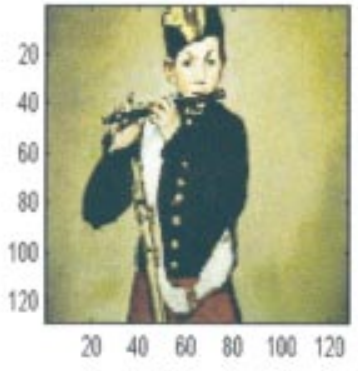

(a)
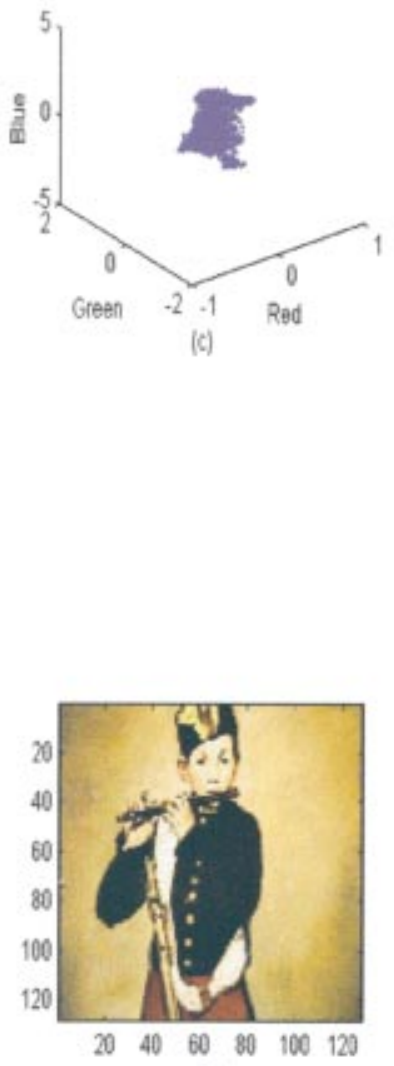

(a)

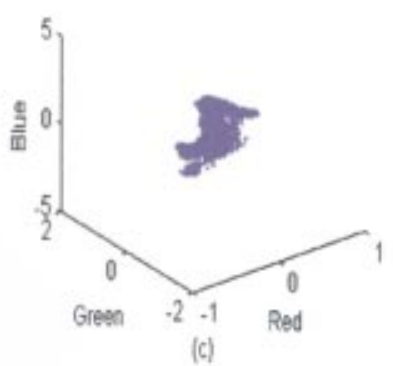

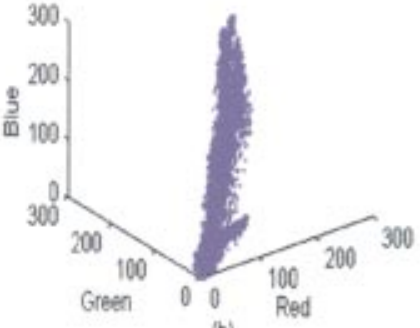

(b)
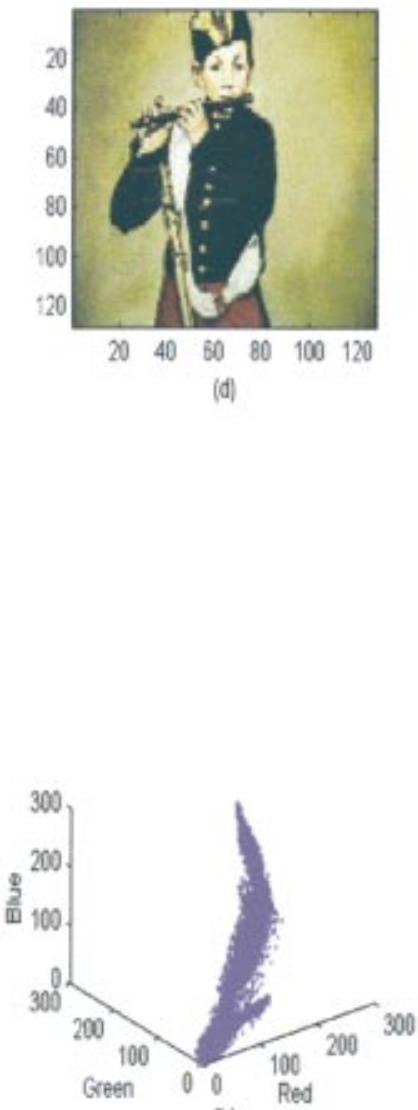

(b)

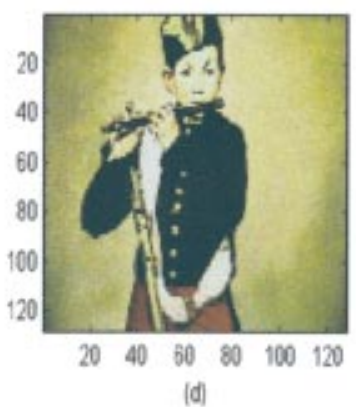

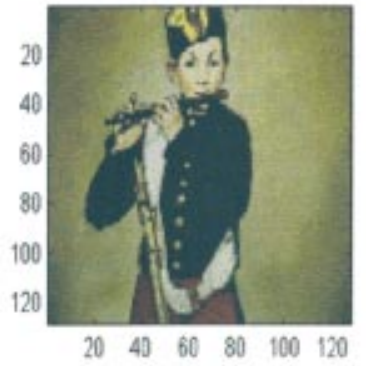

(a)
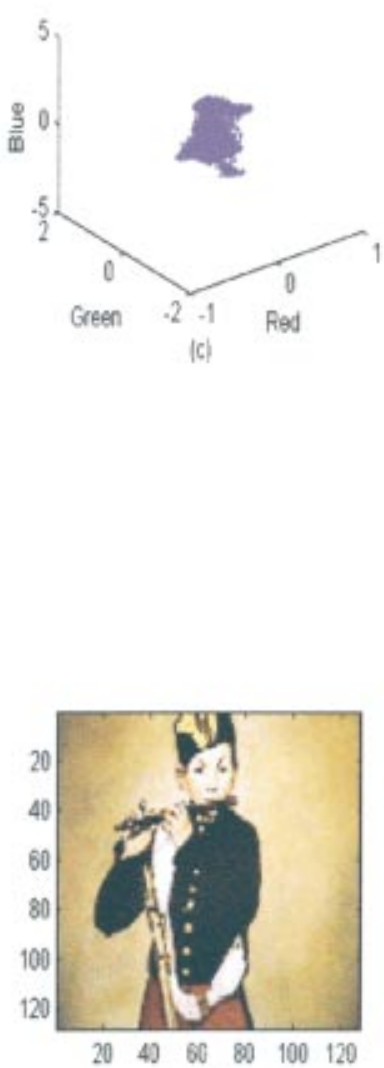

(a)

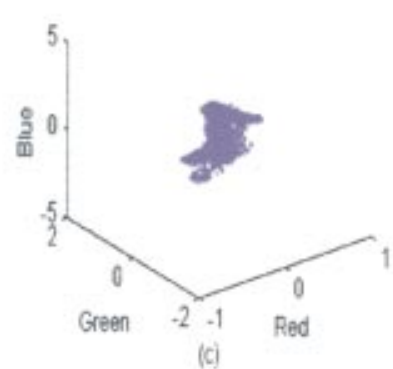

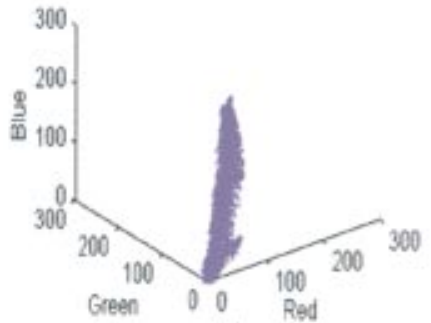

(b)
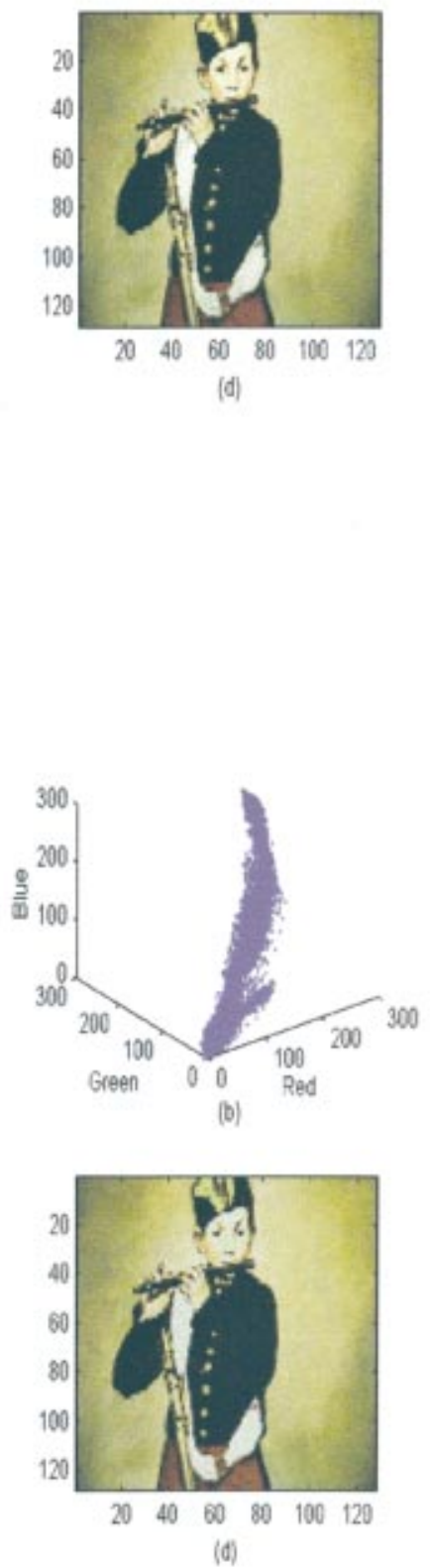

Fig. 3. (a) "Suonatore di Differaro," one original (real) image (top left) taken under standard illumination and three computer-simulated images taken under darker (top right), red (bottom left), and magenta (bottom left) illumination. In each set: (b) color histogram of (a), (c) normalized histogram of (b), (d) recovered image by use of the color histogram normalization algorithm. 
Table 1. PSNR Values of The Basket of Fruit with Computer-Simulated Images (Unit: dB)

\begin{tabular}{lcccc}
\hline Illuminant & PSNR_R & PSNR_G & PSNR_B & $\overline{\text { PSNR }}$ \\
\hline Lighter & 29.6448 & 28.8880 & 31.5366 & 30.0231 \\
Darker & 53.5273 & 53.4203 & 53.5026 & 53.4834 \\
Red & 28.9196 & 39.6264 & 47.6474 & 38.7311 \\
Yellow & 29.1828 & 28.4405 & 40.5630 & 32.7288 \\
Green & 47.2439 & 28.0734 & 47.8528 & 41.0567 \\
Blue & 46.5986 & 39.9857 & 30.5410 & 39.0418 \\
\hline
\end{tabular}

Table 2. PSNR Values of "Suonatore di Differaro" with Computer-Simulated Images (Unit: dB)

\begin{tabular}{lcccc}
\hline Illuminant & PSNR_R & PSNR_G & PSNR_B & $\overline{\text { PSNR }}$ \\
\hline Lighter & 28.3394 & 29.5358 & 36.5034 & 31.4595 \\
Darker & 53.4382 & 53.3757 & 53.1234 & 53.3124 \\
Red & 29.1580 & 33.3205 & 40.3023 & 34.2603 \\
Yellow & 27.5659 & 28.4128 & 34.0331 & 30.0039 \\
Green & 32.3723 & 30.0957 & 36.8836 & 33.1172 \\
Blue & 44.1714 & 42.5236 & 34.6164 & 40.4371 \\
Magenta & 29.7260 & 36.9556 & 36.5139 & 34.3985 \\
\hline
\end{tabular}

Table 3. PSNR Values of "Vaso di Fiori" with Computer-Simulated Images (Unit: dB)

\begin{tabular}{lcccc}
\hline Illuminant & PSNR_R & PSNR_G & PSNR_B & $\overline{\text { PSNR }}$ \\
\hline Lighter & 33.8648 & 29.3721 & 30.4534 & 31.2301 \\
Darker & 53.2074 & 53.3598 & 53.4744 & 53.3472 \\
Red & 31.3609 & 34.9147 & 40.6631 & 35.6462 \\
Yellow & 33.1372 & 28.1924 & 35.3757 & 32.2351 \\
Green & 43.6596 & 28.2873 & 40.5575 & 37.5015 \\
Blue & 40.3268 & 35.4152 & 28.5746 & 34.7722 \\
Magenta & 32.7767 & 40.8628 & 29.6980 & 34.4458 \\
\hline
\end{tabular}

color histogram and the normalized one, respectively, of this test image. There is no need to go into the details of the linear algebra operations; refer to the system diagram Fig. 1. We can obtain a matrix that represents the affine transformation between these two images' histograms. The recovered image is showed in the lower right figure [label (d)]. The rest of Fig. 5 shows the other experiments of the same image under different illumination conditions: yellow, green, and blue. The rest may be deduced by analogy with Fig. 2. Figure 6 shows the same procedure for "Suonatore di Differaro." These images are taken under lighter, darker, red, and magenta different illuminations as the test images to be recovered. In general, the recovery is quite accurate.

The PSNR values of the recovered images in Figs. 5 and 6 are listed in Table 4 and Table 5, respectively. We can obtain approximately $20-30-\mathrm{dB}$ average $\overline{\text { PSNR }}$ for real images. The results for five other images are not displayed here to save space; we list only the PSNR value of "Vaso di Fiori," as shown in Table 6 . Tables 4, 5, and 6 may be compared with Tables 1,2 , and 3, respectively, to find the differences represented by the PSNR's of the computer-simulated images.

\section{Characteristics of the Color Histogram Based on Its Appearance}

From these figures, either computer-simulated images or real images, we can easily find that the position, size, and shape of the color distribution in R-G-B space depends on changes in illumination conditions. For example, for images taken under lighter illuminant, the range of the distribution becomes larger, i.e., the values of R-G-B space are larger; similarly, the range of the distribution becomes smaller for images taken under darker illuminant. Under green illuminant, the position and shape of the distribution are deflected slightly toward the $\mathrm{G}$ axis; i.e., the values of the green band are large; and the position and shape of the distribution are deflected slightly toward the R-G axis for images taken under yellow illuminant.

We found a significant difference between the results of the computer-simulated images and the results of the real images as given in the PSNR tables. For the computersimulated images, when the images are fabricated under green illuminant, the performance of the green band of recovered images is worse; in other words, the value of PSNR_G is smaller, and so forth. Conversely, for real images, when the images are taken under green illuminant, the performance of the green band of recovered images is better; in other words, the value of PSNR_G is larger, and so forth. On the other hand, when the images are taken under darker illuminant, the performance of the three bands of recovered images is nearly the same for all cases. Under yellow illuminant, the performance of the red and green bands may be better than the blue band in the realimage cases, but the result is just the opposite for computer-simulated images. Some reasons for these phenomena are, for example, for the real images taken under red illuminant, the red component of the images is emphasized but the both green and blue components are attenuated. However, for the computer-simulated images, the red component of the images is emphasized but both green and blue components remain unchanged. This may be the reason that the performance of the red band of the recovered image is better for the real images but is worse for the computer-simulated images.

\section{Discussion of Image-Recovery Performance}

For computer-simulated images, the recovered images are quite close to the standard template images; the recovery performance reaches $30 \mathrm{~dB}$ and upward. This is because the noise in the computer-simulated images is uniform and much less than in the real images. However, for practical real-image experiments, although the color distortion of the recovered images cannot be removed entirely, as it can be for computer-simulated images, the degree of color distortion has been reduced greatly. In comparison with the standard template image, the color change corresponding to illumination change clearly us much improved and considerably smooth. The reason that the recovered images of the real images are not as good as those of the computer-simulated images is that there is nonlinear distortion in color in the real images. In general, the $20-\mathrm{dB}$ recovery performance is regarded as good recovery for practical real-image experiments, for human viewing. 


\section{E. Why the Simplified Affine Model Is Preferred to the General Affine Model}

1. Histogram Matching Between the Two Affine Models Now we will demonstrate that the color histogram algorithm considered in the proposed simplified affine model (with respect only to translation, scaling, and rotation) is better than the algorithm considered in a general affine model for illumination-varying applications. In Fig. 7 we can see that the color histogram of the recovered image with use of the proposed affine model is $9 \mathrm{~dB}$ higher in $\overline{\mathrm{PSNR}}$ than with the general affine model and closer to the color histogram of the standard image. In Figs. 7(g) and $7(\mathrm{~h})$, the red points indicate the color histogram of the standard image, the blue points indicate the color histogram of the recovered image, and the black points indicate the overlap between the two parts. Obviously, the overlap in Fig. 7(g) is larger than that in Fig. 7(h), so the assumption that the color histogram algorithm considered in the proposed affine model is better than in a general affine model for the illumination varying application, is supported again.

\section{Comparison of Numerical Affine Parameters of the} Two Affine Models

On the other hand, by using the recent method of Refs. 23 and 24 , we can normalize the $3 \mathrm{D}$ color histogram under general affine distortions. The affine matrices can be estimated very accurately in noise-free cases. ${ }^{23}$ As shown as Fig. 8, we can find that the results of applying the simpli- fied affine model and the general affine model to estimate affine matrices under general affine distortions in noisefree cases are all very precise. So both the simplified affine model and the general affine model are feasible in noise-free cases for simulated illumination-change applications. Note that the $0.1-\mathrm{dB}$ difference in the PSNR is perhaps due to the quantized truncation error during computer calculation. At the same time, we find that the range of the estimated shearing parameter on each axis is between \pm 2 in the color histogram, whether for computersimulated images or for real images. From our experiments the range of the values between \pm 4 does not affect the results of the estimated affine matrix. So the effect of shearing can be neglected in image recovery for illumination-varying applications. As for the skew effect of the color histogram due to illumination changes, we find that the ratios of each eigenvalue of each axis between the standard image and the illumination-change images are almost constant, whether for computersimulated images or for real images. This indicates that the shape of the color histogram does not change dramatically, so the assumption to neglect the effect of skew is reasonable.

\section{Comparison of Image-Recovery Performance of the} Two Affine Models

From the experimental results of the PSNR, as shown in Tables 7-9, we can see the difference between the two
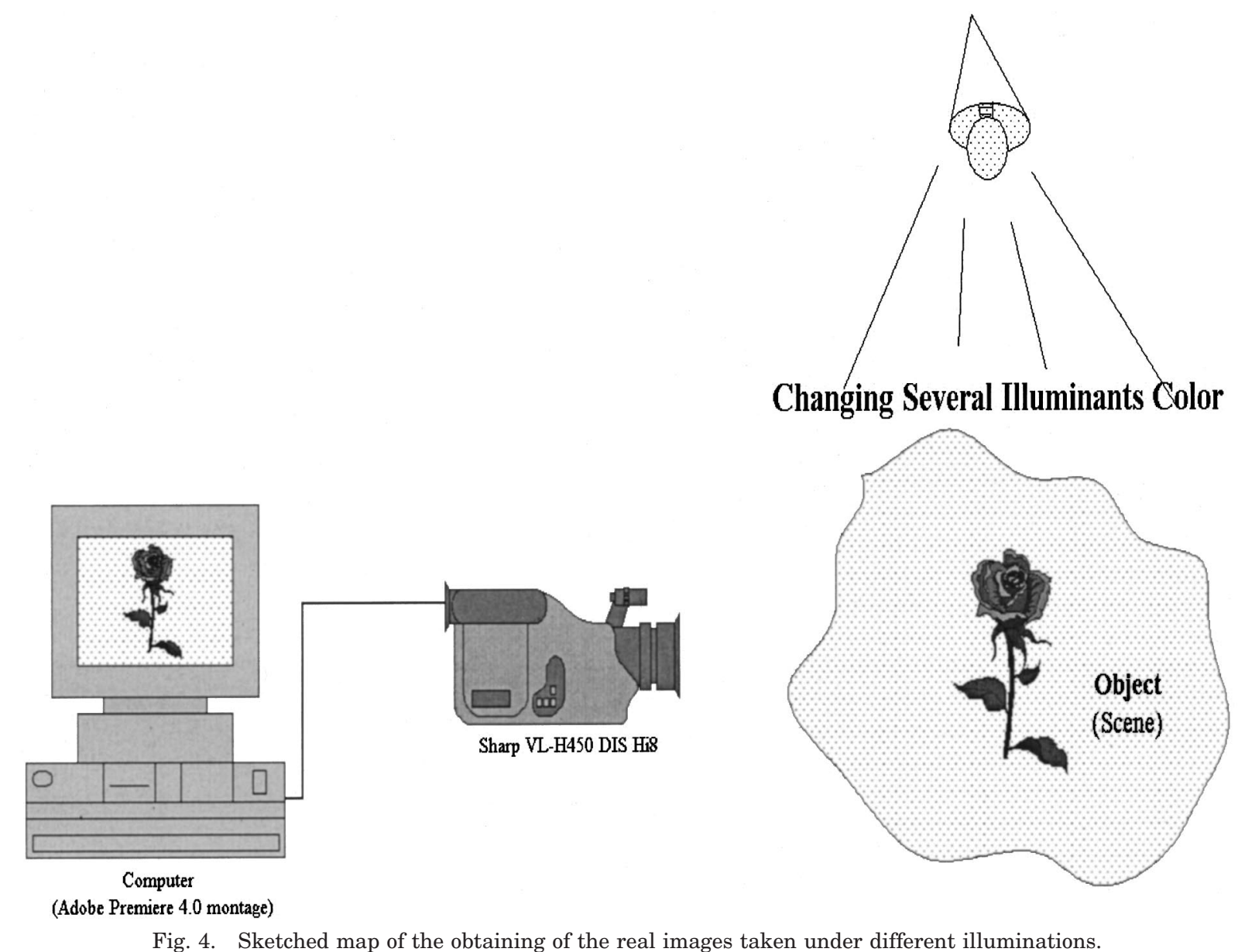

Fig. 4. Sketched map of the obtaining of the real images taken under different illuminations. 

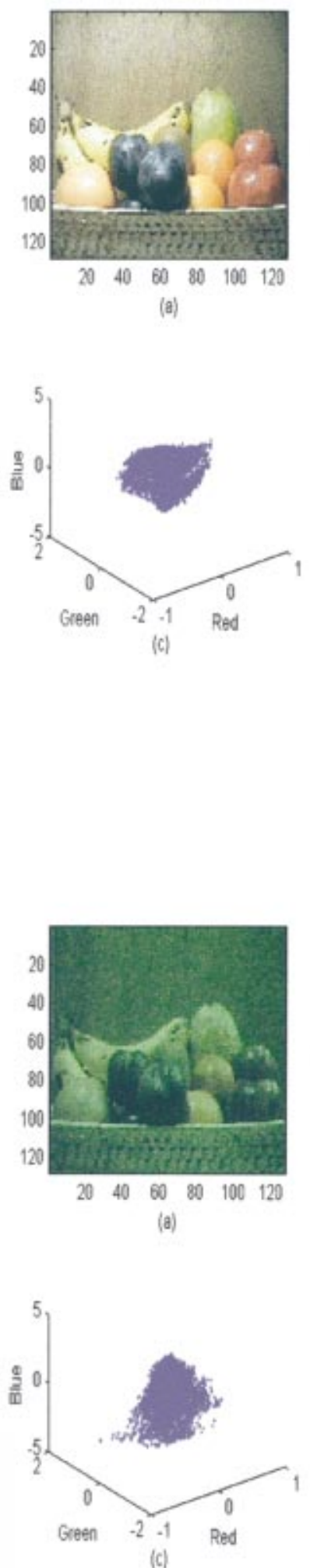
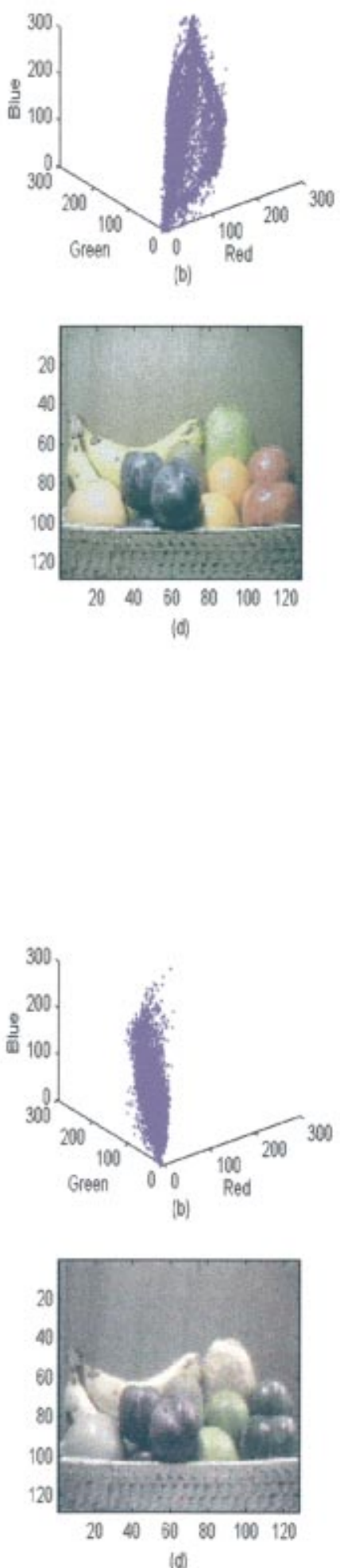
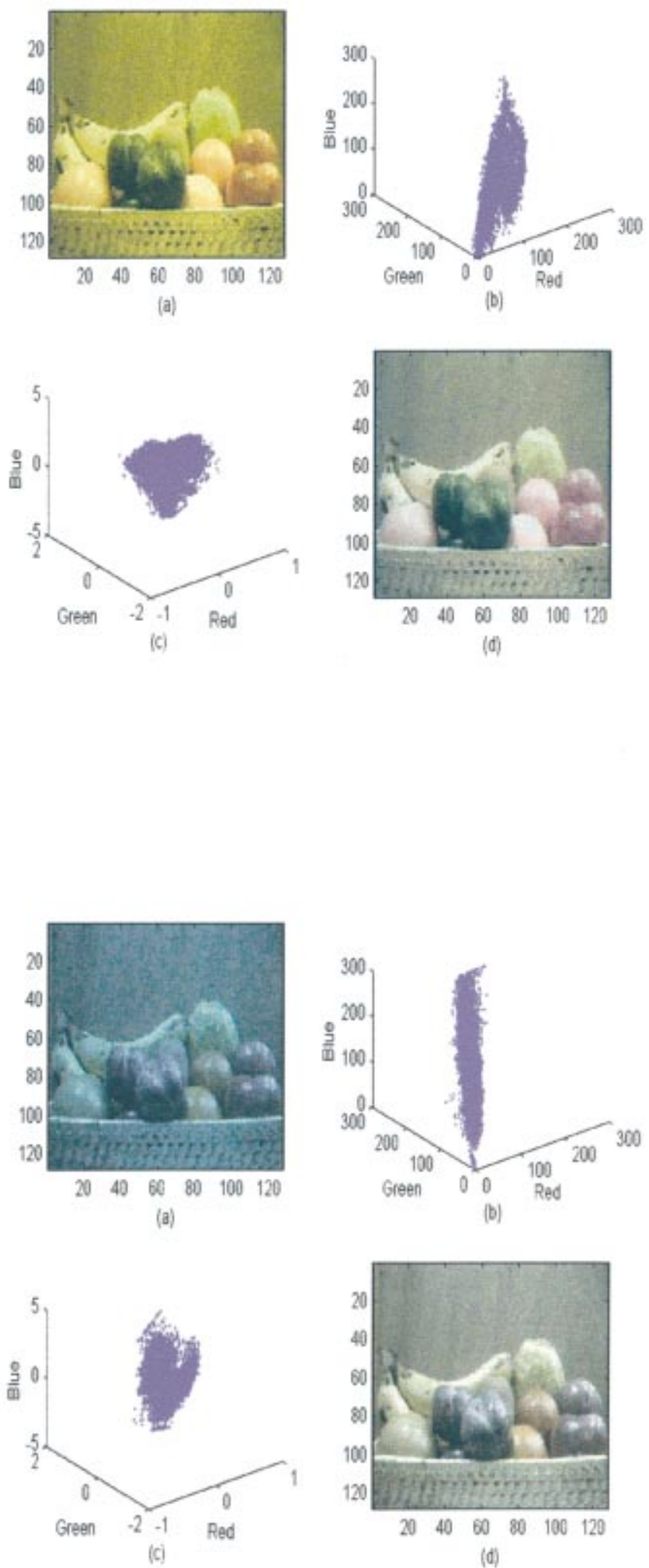

Fig. 5. (a) Basket of fruit, four real images taken under lighter (top left), yellow (top right), green (bottom left), and blue (bottom right) illumination. In each set: (b) color histogram of (a), (c) normalized histogram of (b), (d) recovered image by use of the color histogram normalization algorithm.

models clearly. For three real images, the $\overline{\mathrm{PSNR}}$ of each recovered image with use of the general affine model is 4-5 dB lower than that of the proposed simplified affine model on average, or even lower-11 dB. It may be that the $\overline{\mathrm{PSNR}}$ is lower when the color histogram normalization algorithm is considered in the general affine model for noisy real images because of the problem of noise sensitivity when we estimate the affine matrix with respect 
to the coefficients of the skew. That is, when we intend to solve the effect of skew in the general affine model, we must determine three rotation angles in the normalized color histogram corresponding to orthogonal eigenvectors; but estimations of the three rotation angles are noise sensitive and can easily affect the accuracy of the whole color histogram normalization algorithm. With the general affine model, unlike with simplified algorithm, we consider

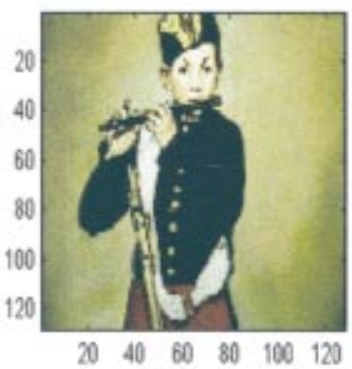

(a)

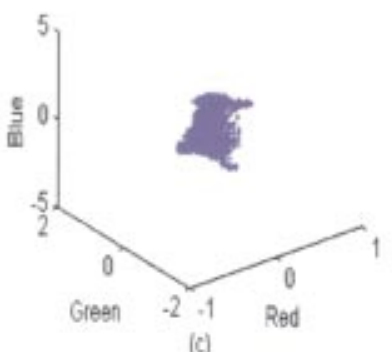

(c)
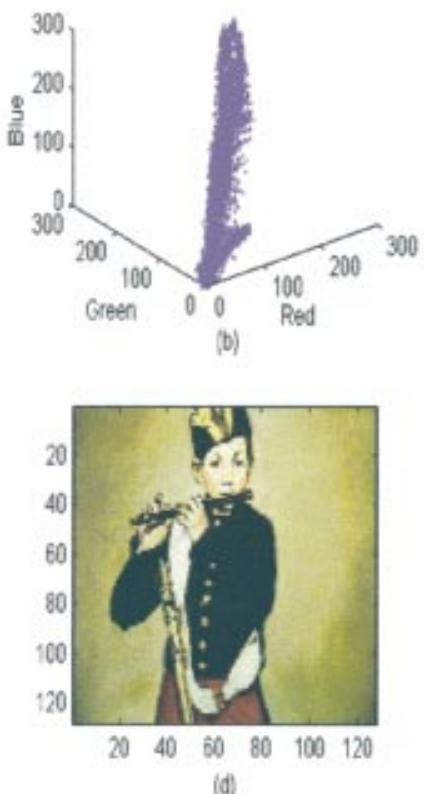

(d)

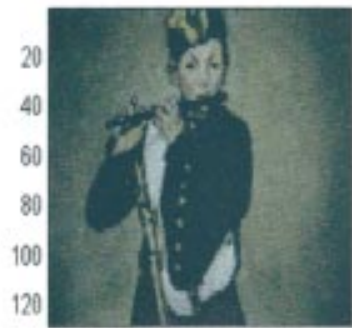

$\begin{array}{llllll}20 & 43 & 60 & 80 & 100 & 120\end{array}$

(a)
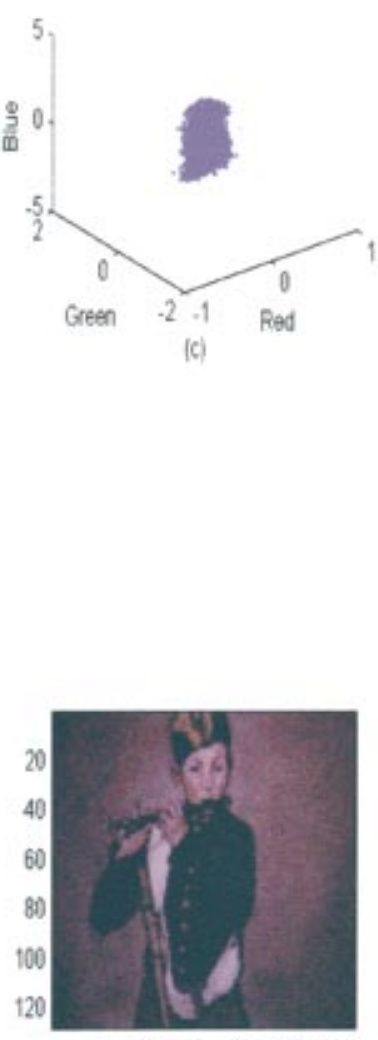

$\begin{array}{lllllll}20 & 40 & 60 & 80 & 100 & 120\end{array}$

(a)

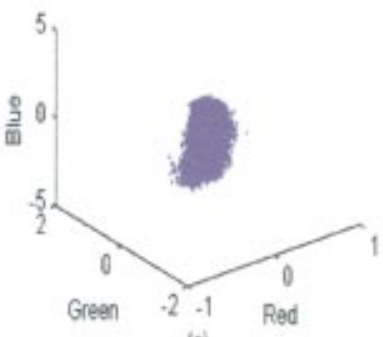

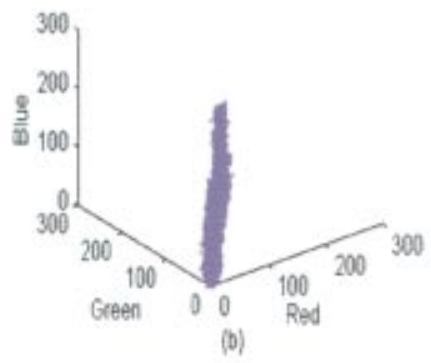
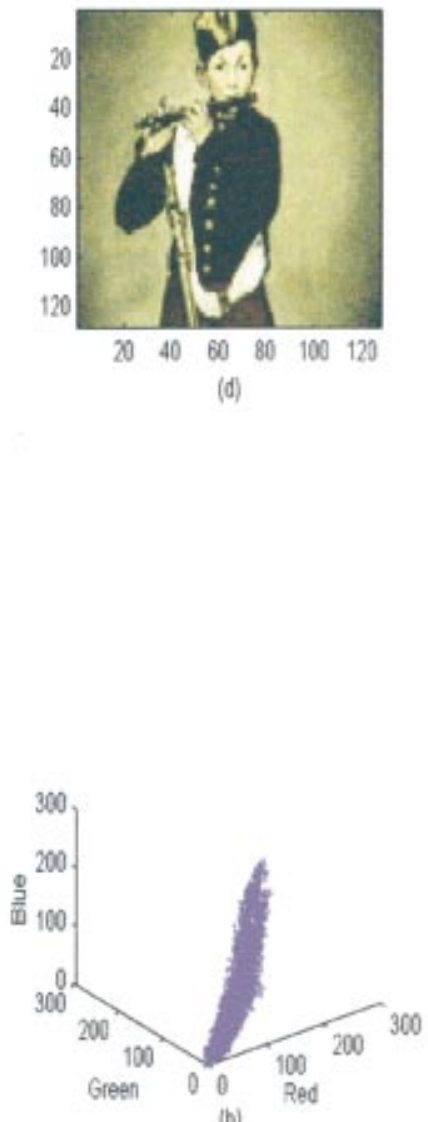

$(b)$

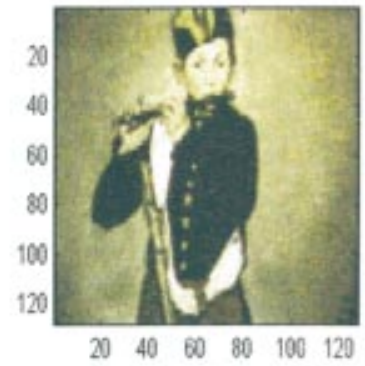

(d)

Fig. 6. (a) "Suonatore di Differaro," four real images taken under lighter (top left), darker (top right), red (bottom left), and magenta (bottom right) illumination. In each set: (b) color histogram of (a), (c) normalized histogram of (b), (d) recovered image by use of the color histogram normalization algorithm. 
Table 4. PSNR Values of The Basket of Fruit with Real Images (Unit: dB)

\begin{tabular}{lcccc}
\hline Illuminant & PSNR_R & PSNR_G & PSNR_B & $\overline{\text { PSNR }}$ \\
\hline Lighter & 18.6641 & 18.4261 & 17.8034 & 18.2980 \\
Darker & 21.0168 & 21.7757 & 21.1011 & 21.2979 \\
Red & 23.3661 & 19.2069 & 17.3586 & 19.9772 \\
Yellow & 25.9697 & 28.6324 & 19.8970 & 24.8330 \\
Green & 21.1517 & 25.0523 & 22.1846 & 22.7962 \\
Blue & 21.5079 & 25.1744 & 27.9541 & 24.8788 \\
\hline
\end{tabular}

Table 5. PSNR Values of "Suonatore di Differaro" with Real Images (Unit: dB)

\begin{tabular}{lcccc}
\hline Illuminant & PSNR_R & PSNR_G & PSNR_B & $\overline{\text { PSNR }}$ \\
\hline Lighter & 32.0487 & 32.8700 & 30.2986 & 31.7391 \\
Darker & 22.2110 & 24.0862 & 24.3619 & 23.5531 \\
Red & 23.6601 & 23.0507 & 20.7801 & 22.4970 \\
Yellow & 29.4005 & 30.8958 & 22.1842 & 27.4935 \\
Green & 25.4368 & 27.0449 & 22.5219 & 25.0012 \\
Blue & 24.2970 & 25.8839 & 25.7457 & 25.3089 \\
Magenta & 20.5472 & 21.0030 & 23.7332 & 21.7612 \\
\hline
\end{tabular}

Table 6. PSNR Values of "Vaso di Fiori" with Real Images (Unit: dB)

\begin{tabular}{lrrrc}
\hline Illuminant & PSNR_R & PSNR_G & PSNR_B & $\overline{\text { PSNR }}$ \\
\hline Lighter & 28.5373 & 29.1762 & 28.4016 & 28.7050 \\
Darker & 22.7644 & 24.6875 & 24.0188 & 23.8235 \\
Red & 23.1677 & 18.2578 & 14.2002 & 18.5426 \\
Yellow & 28.3944 & 22.5648 & 18.1098 & 23.0230 \\
Green & 21.2094 & 26.6574 & 19.3652 & 22.4107 \\
Blue & 19.7930 & 22.3820 & 27.1819 & 23.1189 \\
Magenta & 19.7719 & 20.5447 & 24.2883 & 21.5350 \\
\hline
\end{tabular}

only the reflection of the normalized color histogram and apply tensor theories to solve the problem without using skew rotation. We think that is why the $\overline{\mathrm{PSNR}}$ is lower when the color histogram normalization algorithm is considered in the general affine model. It is worth mentioning that when we perceive the variation of the color histogram due to illumination-change directly through the senses, we find that the color histogram is always within first octant in R-G-B color space whatever the illumination changes may be. Hence an affine model that corresponds to such affine transformations should be a limited and restricted affine model, and the simplified affine model conforms to these conditions.

\section{F. Discussion of Other Factors Not Considered}

As is well known, the difficulties in studying color constancy come from many natural factors, including shadow, illumination conditions, and specular reflection. ${ }^{5}$ Here we have focused on illumination color; shadow and specular reflective effects have not been considered here. Maybe this is the reason that the method does not always work as well for some real images. It should be mentioned that in the color subtractive system the yellow dye acts as a variable absorber of blue light, and the magenta dye acts as a variable absorber of green light. This means that the images taken under yellow illumination can be regarded as images taken under red and green, two different illuminations at the same time; similarly, the magenta illumination can be regarded as the combination of red and blue illuminations. Therefore, if the recovery performance of the image made under yellow or magenta illumination is good, it can be expected that the result should be good when the image is illuminated by two different illuminations at the same time. Finally, we emphasize that because the effect of translation, scale, and rotation are dominant in the appearance of the color histogram corresponding to the illumination changes, we have discussed only these three cases of the color histogram in this paper.

\section{CONCLUSIONS}

Many researchers have used color information to recognize objects in recent years, but the color of an object varies with changes in illumination source, illumination geometry, viewing angle, and miscellaneous sensor parameters. Therefore normalization of the color distribution of observed images is an important task for image recovery. In this paper we have proposed a novel color histogram normalization by use of the proposed simplified affine model to recover color images. The algorithm uses the statistical properties (covariance matrix) of the color histogram in R-G-B coordinate and tensor theories to achieve image normalization. Then, comparing the normalized color histogram of the standard image with the that of the tested image and performing some operations of simple linear algebra, we estimated the matrix of affine transformation between two images under different illuminations. The image recovered was close to the standard image with use of the estimated affine transformation. The experiment was divided into two parts to test the performance of the proposed algorithm in different circumstances. In the first experiment we applied image-processing software to simulate the illuminantchanged images; we also obtained real images, taken under different illuminations by a camera. From the experimental results, for both computer-simulated images and real images, we found that most of recovered images were quite close to the original images, in particular, the computer-simulated images were less noisy than the real images. In other words, the recovery performance was quite successful and satisfactory for both cases. We explained the noise-sensitive skew-rotation estimation with the general affine model, and we demonstrated that the proposed simplified affine model without using skew rotation is better than the general affine model for such applications. 


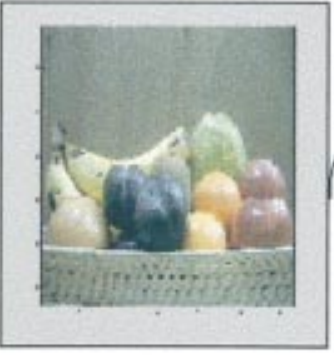

(a)

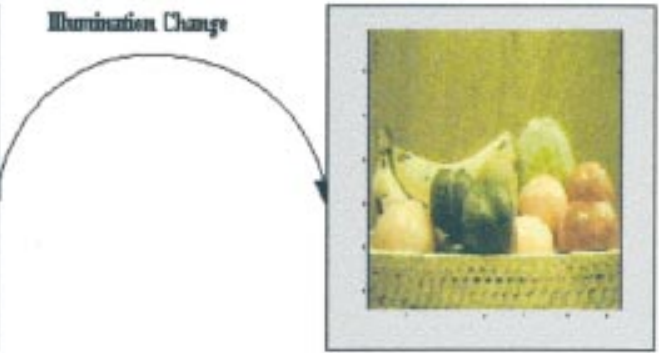

(b)

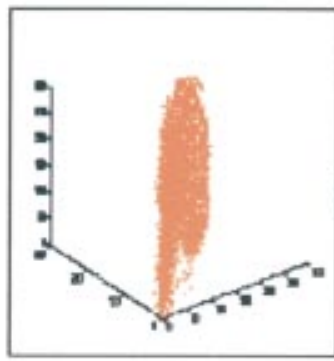

(c)
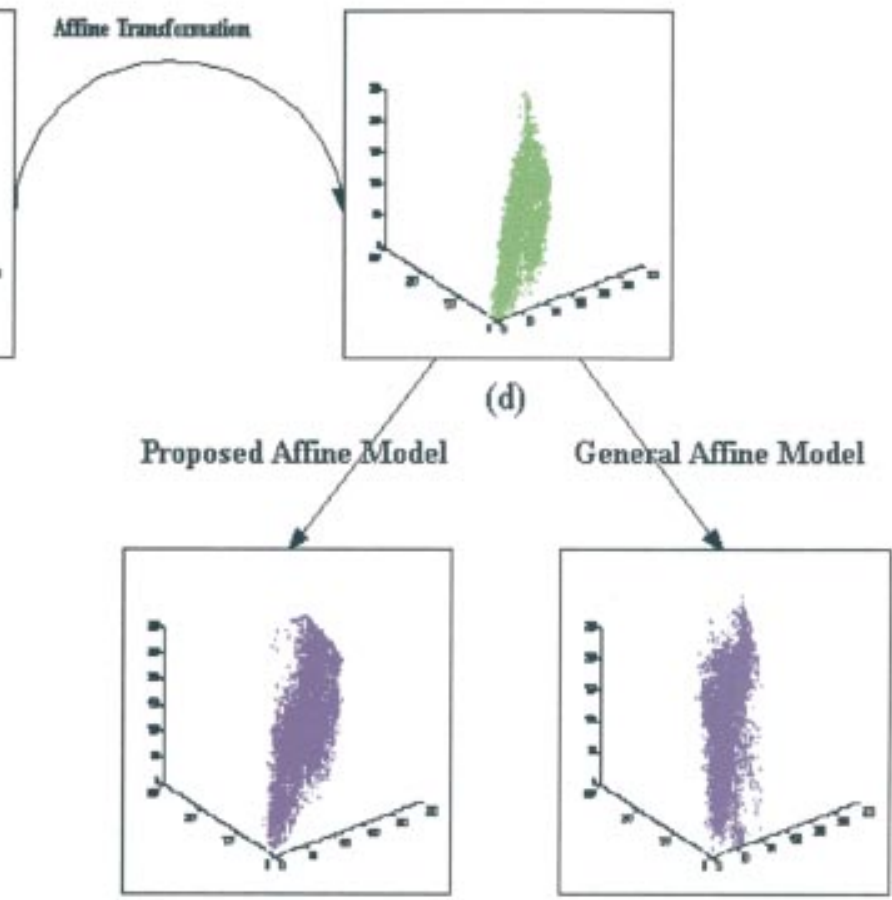

(c)

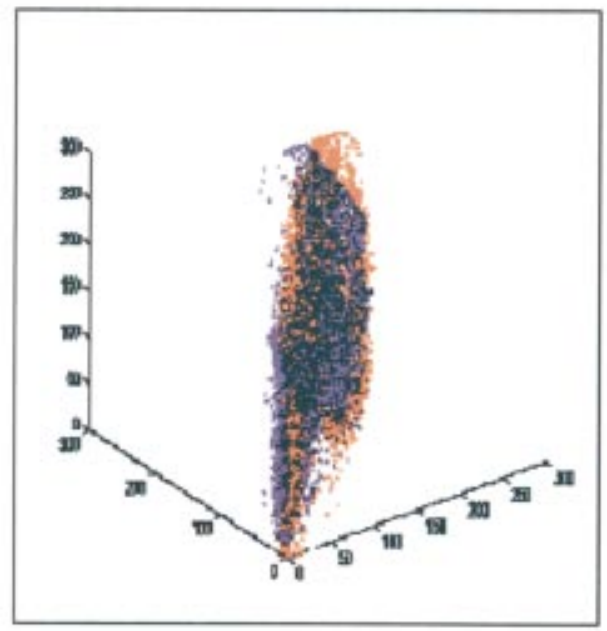

(g)

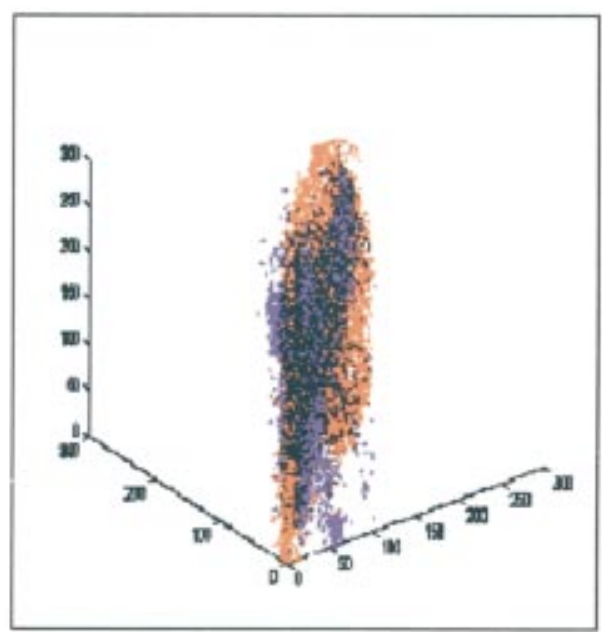

(h)

Fig. 7. Comparison of the color histogram normalization algorithm with two different affine models: (a) original image, (b) real image (under yellow illumination), (c) color histogram of (a),(d) color histogram of (b), (e) color histogram of the recovered image whose algorithm is considered in the proposed simplified affine model, (f) color histogram of the recovered image whose algorithm is considered in the general affine model, (g) color histogram of the companison between the original image and the recovered image by use of the proposed simplified affine model, (h) color histogram of the comparison between the original image and the recovered image by use of the general affine model. The PSNR of (e) is $\sim 9 \mathrm{~dB}$ higher than that of (f). 


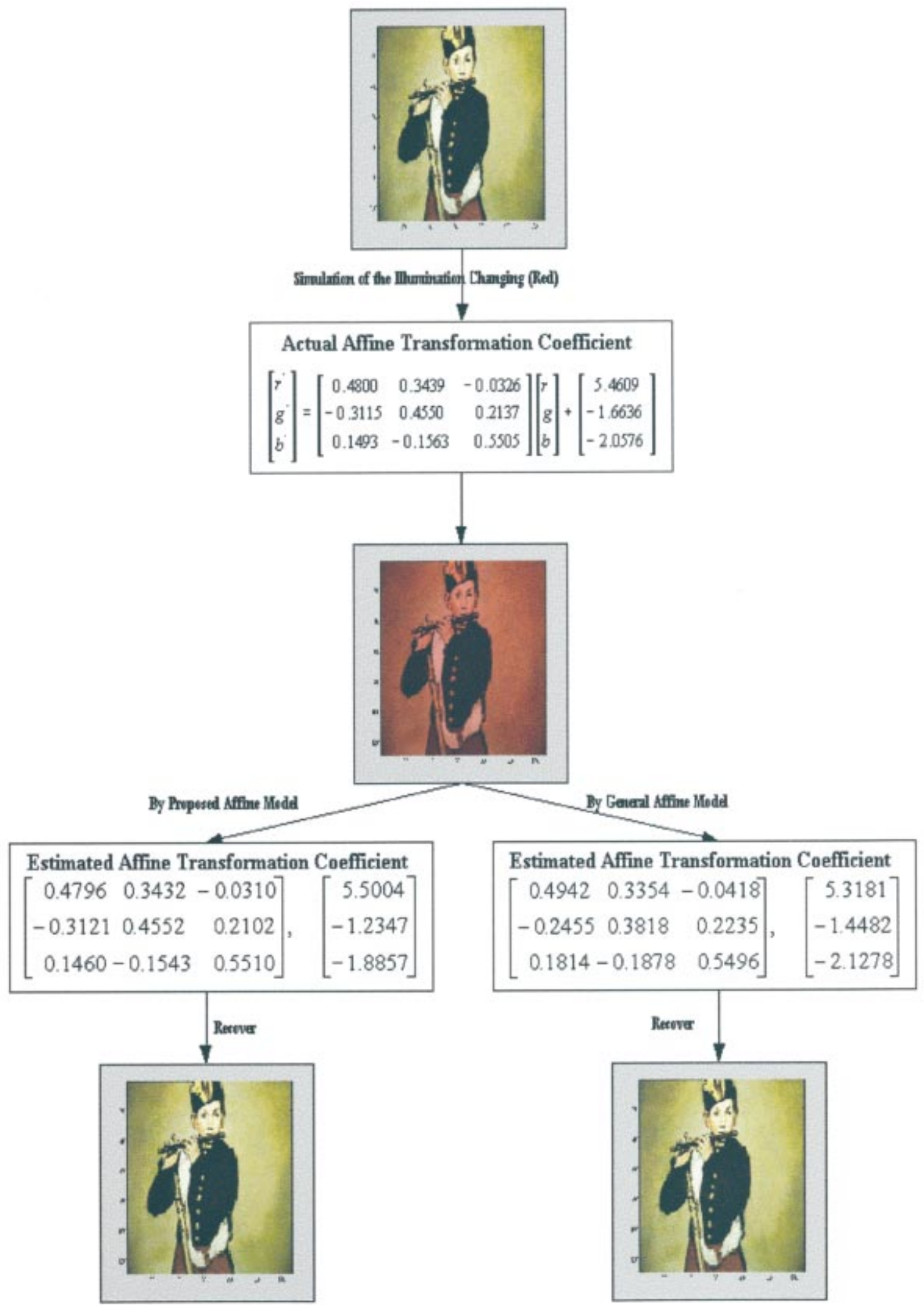

Fig. 8. Comparison of the difference in the estimation of the affine transformation coefficient by the simplified affine model and the general affine model. It can be seen that the former is a little more accurate than the latter. So the estimation of the coefficient by using either simplified affine model or general affine model is feasible for noise-free case of such illuminant-changing application. Note that $\overline{\text { PSNR }}$ of recovered image by using color histogram normalization algorithm considered in the simplified affine model is 47.8546 , and $\overline{\mathrm{PSNR}}$ of recovered image considered in the general affine model is 47.7566 ; the difference $0.1 \mathrm{~dB}$ maybe is due to the quantized truncation error during computer calculation. 
Table 7. Comparison of $\overline{\text { PSNR Values of }}$ "The Basket of Fruit" with Two Different Affine Models (Real Image)

\begin{tabular}{lcc}
\hline$\overline{\text { PSNR }(\mathrm{dB})}$ & Proposed & General \\
\hline Lighter & 18.2980 & 17.9645 \\
Darker & 21.2979 & 14.9077 \\
Red & 19.9772 & 14.1945 \\
Yellow & 24.8330 & 15.1205 \\
Green & 22.7962 & 13.8863 \\
Blue & 24.8788 & 17.5003 \\
\hline
\end{tabular}

Table 8. Comparison of PSNR Values of "Suonatore di Differaro" with Two Different Affine Models (Real Image)

\begin{tabular}{lcc}
\hline$\overline{\text { PSNR }(\mathrm{dB})}$ & Proposed & General \\
\hline Lighter & 31.7391 & 30.0313 \\
Darker & 23.5531 & 17.9176 \\
Red & 22.4970 & 11.3853 \\
Yellow & 27.4935 & 23.7306 \\
Green & 25.0012 & 18.1193 \\
Blue & 25.3089 & 17.3467 \\
Magenta & 21.7612 & 18.2988 \\
\hline
\end{tabular}

Table 9. Comparison of $\overline{\text { PSNR }}$ Values of "Vaso di Fiori" with Two Different Affine Models (Real Image)

\begin{tabular}{lcc}
\hline$\overline{\text { PSNR }}(\mathrm{dB})$ & Proposed & General \\
\hline Lighter & 28.7050 & 16.9603 \\
Darker & 23.8235 & 18.5292 \\
Red & 18.5426 & 12.3300 \\
Yellow & 23.0230 & 20.0113 \\
Green & 22.4107 & 13.8471 \\
Blue & 23.1189 & 16.1045 \\
Magenta & 21.5350 & 12.3004 \\
\hline
\end{tabular}

S.-C. Pei can be reached at the address on the title page or by phone, 886-2-2363-5251, ext. 321; fax, 886-2-23671909; or e-mail, pei@cc.ee.ntu.edu.tw.

\section{REFERENCES}

1. M. Swain and D. Ballard, "Color indexing," Int. J. Comput. Vision 7, 11-32 (1991).

2. B. Funt and G. Finlayson, "Color constant color indexing," IEEE Trans. Pattern Anal. Mach. Intell. 17, 522-529 (1995).

3. B. K. P. Horn, Robot Vision (MIT Press, Cambridge, Mass., 1987).

4. L. Maloney and B. Wandell, "Color constancy: a method for recovering surface spectral reflectance," J. Opt. Soc. Am. A 3, 29-33 (1986).

5. S. Lin and S. W. Lee, "Using chromaticity distributions and eigenspace analysis for pose-, illumination-, and specularity-invariant recognition of 3D objects," in Proceed- ings of the 1997 IEEE Computer Society Conference on Computer Vision and Pattern Recognition (IEEE Computer Society, Los Alamitos, Calif., 1997), pp. 426-431.

6. E. H. Land and J. J. McCann, "Lightness and retinex theory," J. Opt. Soc. Am. 61, 1-11 (1971).

7. M. D'Zmura and P. Lennie, "Mechanisms of color constancy,” J. Opt. Soc. Am. A 3, 1662-1672 (1986).

8. G. Healey, "Estimating spectral reflectance using highlights," Image Vision Comput. 9, 333-337 (1991).

9. D. Slater and G. Healey, "The illumination-invariant matching of deterministic local structure in color images," IEEE Trans. Pattern Anal. Mach. Intell. 19, 1146-1151 (1997).

10. G. Healey and D. Slater, "Global color constancy: recognition of objects by use of illumination-invariant properties of color distributions," J. Opt. Soc. Am. A 11, 3003-3010 (1994).

11. D. Slater and G. Healey, "The illumination-invariant recognition of 3D objects using local color invariants," IEEE Trans. Pattern Anal. Mach. Intell. 18, 206-210 (1996).

12. G. Healey and D. Slater, "Computing illuminationinvariant descriptors of spatially filtered color image regions," IEEE Trans. Image Process. 6, 1002-1013 (1997)

13. L. Maloney, "Evaluation of linear models of surface spectral reflectance with small numbers of parameters," J. Opt. Soc. Am. A 3, 1673-1683 (1986).

14. J. P. S. Parkkinen, J. Hallikainen, and T. Jaaskelainen, "Characteristic spectra of Munsell colors," J. Opt. Soc. Am. A 6, 318-322 (1989).

15. B. Wandell, "The synthesis and analysis of color images," IEEE Trans. Pattern Anal. Mach. Intell. PAMI-9, 2-13 (1987).

16. J. Cohen, "Dependency of the spectral reflectance curves of the Munsell color chips," Psychon. Sci. 1, 369-370 (1964).

17. I. Rothe, K. Voss, and H. Suesse, "The method of normalization to determine invariants," IEEE Trans. Pattern Anal. Mach. Intell. 18, 366-376 (1996).

18. D. Paulus, L. Csink, and H. Niemann, "Color cluster rotation," in Proceedings of the 1998 IEEE International Conference on Image Processing (Institute of Electrical and Electronics Engineers, New York, 1998), pp. 161-165.

19. S. C. Pei and C. N. Lin, "Image normalization for pattern recognition," Image Vision Comput. 13, 711-723 (1995).

20. D. Cyganski and J. A. Orr, "Application of tensor theory to object recognition and orientation determination," IEEE Trans. Pattern Anal. Mach. Intell. PAMI-7, 662-673 (1985).

21. E. Oja, Subspace Methods for Pattern Recognition (Research Studies Press, Letchworth, Hertfordshire, England, 1983).

22. E. Gose, R. Johnsonbaugh, and S. Jost, Pattern Recognition and Image Analysis (Prentice-Hall PTR, Upper Saddle River, N.J., 1996).

23. S. C. Pei and C. L. Tseng, "Illuminant-invariant color image recognition using color histogram normalization and block average feature," manuscript available from the authors.

24. G. Bebis, M. Georgiopoulos, N. da Vitoria Lobo, and M. Shah, "Learning affine transformations of the plane for model-based object recognition," in Proceedings of the 13th International Conference on Pattern Recognition (Institute of Electrical and Electronics Engineers, New York, 1996), Vol. 4, pp. 60-64.

25. C. C. Hung, A. Fahsi, W. Tadesse, and T. Coleman, "A comparative study of remotely sensed data classification using principal components analysis and divergence," in Proceedings of the 1997 IEEE International Conference on Systems, Man, and Cybernetics (Institute of Electrical and Electronics Engineers, New York, 1997), Vol. 3, pp. 2444-2449.

26. A. Z. Kouzani, F. He, and K. Sammut, "Quadtree principal component analysis and its application to facial expression classification," in Proceedings of the 1999 IEEE International Conference on Systems, Man, and Cybernetics (Institute of Electrical and Electronics Engineers, New York, 1999), Vol. 6, pp. 835-839. 
27. T. Kawatani, "Handwritten kanji recognition using combined complementary classifiers in a cascade arrangement," in Proceedings of the Fifth International Conference on Document Analysis and Recognition (Institute of Electrical and Electronics Engineers, New York, 1999), pp. 503-506.

28. S. C. Pei and C. C. Wu, "Normalization of 3-D objects under general affine transformations," manuscript available from the authors.

29. C. C. $\mathrm{Wu}$, "2-D Images and 3-D objects normalization using moment-based covariance matrices" (Master's Thesis, Graduate Institute of Communication Engineering, National Taiwan University, Taipei, Taiwan, 2000). 